Article

\title{
Pythagorean Fuzzy Muirhead Mean Operators and Their Application in Multiple-Criteria Group Decision-Making
}

\author{
Jianghong Zhu ${ }^{1, *}$ and Yanlai Li ${ }^{1,2}$ \\ 1 School of Transportation and Logistics, Southwest Jiaotong University, Chengdu 611756, China; \\ yanlaili@home.swjtu.edu.cn or yanlaili2010@163.com \\ 2 National Laboratory of Railway Transportation, Southwest Jiaotong University, Chengdu 611756, China \\ * Correspondence: zhujianghong007@163.com
}

Received: 25 May 2018; Accepted: 7 June 2018; Published: 11 June 2018

\begin{abstract}
As a generalization of the intuitionistic fuzzy set (IFS), a Pythagorean fuzzy set has more flexibility than IFS in expressing uncertainty and fuzziness in the process of multiple criteria group decision-making (MCGDM). Meanwhile, the prominent advantage of the Muirhead mean (MM) operator is that it can reflect the relationships among the various input arguments through changing a parameter vector. Motivated by these primary characters, in this study, we introduced the MM operator into the Pythagorean fuzzy context to expand its applied fields. To do so, we presented the Pythagorean fuzzy MM (PFMM) operators and Pythagorean fuzzy dual MM (PFDMM) operator to fuse the Pythagorean fuzzy information. Then, we investigated their some properties and gave some special cases related to the parameter vector. In addition, based on the developed operators, two MCGDM methods under the Pythagorean fuzzy environment are proposed. An example is given to verify the validity and feasibility of our proposed methods, and a comparative analysis is provided to show their advantages.
\end{abstract}

Keywords: Pythagorean fuzzy set; Muirhead mean; multiple criteria group decision-making

\section{Introduction}

Multi-criteria group decision-making (MCGDM), a sub-field of decision-making, is a common and important activity in the real world, and is especially useful in the fields of engineering, economic, management, and the military. In practical applications, a critical problem is how to express the valuation information provided by decision makers. Due to the complexity and fuzziness of MCGDM problems, it is difficult for decision makers to give precise valuation information through employing crisp numbers. Fuzzy set (FS) theory, originally developed by Zadeh [1], is a particularly effective tool to capture uncertain and fuzzy information. However, due to the FS having only one membership degree, it cannot deal effectively with some complicated fuzzy information. Therefore, Atanassov and Rangasamy [2] developed intuitionistic fuzzy set (IFS) through introducing the non-membership degree into the FS. In IFS, the sum of the membership degree and non-membership degree needs to be equal to or less than 1 . However, in some practical applications, IFS cannot solve the problem that the sum of the membership and non-membership is bigger than 1, but the square sum is equal to or less than 1. To overcome this drawback of IFS, Pythagorean fuzzy set (PFS), as a generalization of IFS, was introduced by Yager [3,4], of which the square sum of the membership degree and non-membership degree is less than or equal to 1 . In other words, when we treat uncertainty and fuzziness in practical MCGDM problems, PFS is a more effective and flexible tool compared with IFS.

Based on some existing aggregation operators, various aggregation operators of Pythagorean fuzzy set have been developed by a number of researchers to solve multi-criteria decision-making 
(MCDM) problems with Pythagorean fuzzy information. Depending on whether the input argument is independent, these operators can be divided into two categories: (1) the input argument is independent; (2) any two input arguments are correlated. Many operators fall into the former category. For example, Yager [3,4] developed the Pythagorean fuzzy weighted averaging (PFWA) and Pythagorean fuzzy weighted geometric (PFWG) operators, and used these to solve Pythagorean fuzzy MCDM problems. Based on the operational laws proposed by Zhang and $\mathrm{Xu}$ [5], Ma and $\mathrm{Xu}$ [6] presented two new PFWA and PFWG operators, symmetric Pythagorean fuzzy weighted geometric/averaging operators, and examined the relationships between these operators and the operators proposed by Yager. Rahman et al. [7] proposed the Pythagorean fuzzy Einstein weighted geometric operator and discussed its desirable properties and special cases. Garg [8] introduced the Einstein operational laws into the Pythagorean fuzzy environment to develop two generalized averaging aggregation operators, and utilized these operators to solve MCDM problems. Through incorporating the confidence level into each Pythagorean fuzzy number, Garg [9] presented a series of novel averaging and geometric operators. Zeng et al. [10] proposed the Pythagorean fuzzy ordered weighted averaging weighted averaging distance operator. On the other hand, Peng and Yang [11] extended the Choquet integral into the Pythagorean fuzzy environment to propose a Pythagorean fuzzy Choquet integral operator. Wei and $\mathrm{Lu}$ [12] presented some Pythagorean fuzzy power aggregation operators based on the power aggregation operator, and investigated the main characteristics of these operators. Liang et al. [13] developed the Pythagorean fuzzy Bonferroni mean operator and their weighted form. Moreover, some properties and cases of the proposed operators are explored and an accelerative calculating algorithm is designed to simplify the computation process of the presented operators. Liang et al. [14] proposed the Pythagorean fuzzy weighted geometric Bonferroni mean operator and applied it to handle MCGDM problems with Pythagorean fuzzy information. In real decision-making, however, a relationship may exist among more than two input arguments due to the complexity of decision-making problems. Thus it can be seen that it is difficult for the above operators to capture the relationships between three or more Pythagorean fuzzy input arguments.

The Muirhead mean (MM) operator, originally presented by Muirhead [15], is a well-known information fusion operator and provides us with a new fusion method for the correlation information. The primary characteristic of the MM operator is that it can reflect the relationship among any number of input arguments. In addition, some existing operators including the arithmetic and geometric averaging, Bonferroni mean [16] and Maclaurin symmetric mean [17] are special cases of it. Consequently, some researchers have extended the MM operator into various fuzzy environments. For instance, Qin and Liu [18] presented some 2-tuple linguistic MM operators by introducing the MM operator into the 2-tuple linguistic context, and utilized them to solve the supplier selection problems. Liu and You [19] developed some interval neutrosophic MM operators based on the MM operator, and presented two novel approaches to handle multiple attribute group decision-making problems in light of the proposed operators. Liu and Li [20] explored the MM operator under the intuitionistic fuzzy environment, and proposed some intuitionistic fuzzy MM operators. Liu et al. [21] introduced the MM operator into a hesitant fuzzy linguistic environment, and developed a hesitant fuzzy linguistic MM operator and its weighted form. Wang et al. [22] extended the MM operator to a hesitant fuzzy linguistic set, and proposed the hesitant fuzzy linguistic MM operator and hesitant fuzzy linguistic dual MM operator and their weighted forms. Based on the Archimedean t-norm and t-conorm, Liu and Teng [23] put forward some probabilistic linguistic Archimedean MM operators and further explored some special cases. Liu et al. [24] proposed an interval 2-tuple weighted MM operator by enlarging the scope of MM operator to the interval 2-tuple linguistic environment, and applied the proposed operator to present a large group dependence evaluation model for human reliability analysis. When we consider the relationship among any number of input arguments, however, the above operators fail to deal with the Pythagorean fuzzy information.

According to the above analysis, we know that the existing aggregation operators of Pythagorean fuzzy cannot capture the relationships between any number of input arguments in the information 
fusion process. At the same time, the MM operator can reflect the relationships between input arguments, so it is necessary to extend it to handle Pythagorean fuzzy information. Hence, inspired by the ideal characteristics of the MM operator, the present paper aims at developing some new aggregation operators of Pythagorean fuzzy to solve MCGDM problems in which we consider the interrelationship among any number of input arguments.

In order to accomplish this goal, the remainder of this paper is arranged as follows. In Section 2, we describe some basic concepts and operational laws of PFS. Based on the MM operator, we develop the Pythagorean fuzzy MM operator and Pythagorean fuzzy weighted MM operator, and the Pythagorean fuzzy dual MM operator and Pythagorean fuzzy dual weighted MM operator in Sections 3 and 4, respectively. In Section 5, we utilize these operators to present two MCGDM methods for the MCGDM problem with Pythagorean fuzzy information. In Section 6, an example is provided to demonstrate the effectiveness and feasibility of the developed approaches, and the advantages of the proposed operators are illustrated by comparing them with the existing operators. Finally, a brief conclusion and future work directions are given in Section 7.

\section{Preliminaries}

In this section, some fundamental concepts related to the Pythagorean fuzzy number (PFN) are briefly introduced below, which will be used in the following sections.

Definition $1[3,4]$. Let $X=\left\{x_{1}, x_{2}, \cdots, x_{n}\right\}$ be a finite nonempty set, and a PFS P in X is defined as follows

$$
X=\left\{<x, \mu_{P}(x), v_{P}(x)>\mid x \in X\right\}
$$

where $\mu_{P}(x) \in[0,1]$ and $v_{P}(x) \in[0,1]$ are defined as the degree of membership and non-membership of the element $x \in X$ to $P$, respectively, and satisfy $\mu_{P}^{2}(x)+v_{P}^{2}(x) \leq 1$. For every $x \in X$, we designate $\pi_{P}(x)$ as the degree of indeterminacy of the PFS, where $\pi_{P}(x)=\sqrt{1-\mu_{P}^{2}(x)-v_{P}^{2}(x)}$. For convenience, $\alpha=\left(\mu_{P}, v_{P}\right)$ is called as a PFN, and $\mu_{P}^{2}+v_{P}^{2} \leq 1$ and $\pi_{P}=\sqrt{1-\mu_{P}^{2}-v_{P}^{2}}$.

Definition 2 [5]. Let $\alpha_{1}=\left(\mu_{P_{1}}, v_{P_{1}}\right), \alpha_{2}=\left(\mu_{P_{2}}, v_{P_{2}}\right)$ and $\alpha=\left(\mu_{P}, v_{P}\right)$ be three PFNs, and $\lambda>0$. Then the basic operational laws of PFN can be defined as follows:

(1) $\quad \alpha_{1} \oplus \alpha_{2}=\left(\sqrt{\mu_{P_{1}}^{2}+\mu_{P_{2}}^{2}-\mu_{P_{1}}^{2} \mu_{P_{2}}^{2}}, v_{P_{1}} v_{P_{2}}\right)$;

(2) $\quad \alpha_{1} \otimes \alpha_{2}=\left(\mu_{P_{1}} \mu_{P_{2}}, \sqrt{v_{P_{1}}^{2}+v_{P_{2}}^{2}-v_{P_{1}}^{2} v_{P_{2}}^{2}}\right)$;

(3) $\lambda \alpha=\left(\sqrt{1-\left(1-\mu_{P}^{2}\right)^{\lambda}},\left(v_{P}\right)^{\lambda}\right)$;

(4) $\quad \alpha^{\lambda}=\left(\left(\mu_{P}\right)^{\lambda}, \sqrt{1-\left(1-\mu_{P}^{2}\right)^{\lambda}}\right)$.

Definition 3 [25]. Let $\alpha=\left(\mu_{P}, v_{P}\right)$ be a PFN, then the score and accuracy function of $\alpha$ is defined respectively as follows

$$
\begin{gathered}
S(\alpha)=\frac{1}{2}\left(1+\mu_{P}^{2}-v_{P}^{2}\right), \\
H(\alpha)=\mu_{P}^{2}+v_{P}^{2} .
\end{gathered}
$$

Definition 4 [25]. Let $\alpha=\left(\mu_{P_{1}}, v_{P_{1}}\right)$ and $\beta=\left(\mu_{P_{2}}, v_{P_{2}}\right)$ be any two PFNs, $S(\alpha)$ and $H(\alpha)$ be the score and accuracy function of $\alpha$, and $S(\beta)$ and $H(\beta)$ be the score and accuracy function of $\beta$, then

(1) If $S(\alpha)>S(\beta)$, then $\alpha$ is superior to $\beta, \alpha>\beta$;

(2) If $S(\alpha)=S(\beta)$, then

(a) If $H(\alpha)>H(\beta)$, then $\alpha$ is superior to $\beta, \alpha>\beta$; 
(b) If $H(\alpha)=H(\beta)$, then $\alpha$ is equivalent to $\beta \alpha=\beta$.

Definition 5 [4,5]. Let $\alpha_{1}=\left(\mu_{P_{1}}, v_{P_{1}}\right)$ and $\alpha_{2}=\left(\mu_{P_{2}}, v_{P_{2}}\right)$ be two PFNs, the ordering relationship on the PFNs is defined as follows: $\alpha_{1} \geq \alpha_{2}$ if and only if $\mu_{P_{1}} \geq \mu_{P_{2}}$ and $\nu_{P_{1}} \leq v_{P_{2}}$.

\section{Some Pythagorean Fuzzy Muirhead Operators}

The Pythagorean fuzzy MM (PFMM) operator and Pythagorean fuzzy weighted MM (PFWMM) operator are defined in Sections 3.1 and 3.2, respectively.

\subsection{The PFMM Operator}

Definition 6 [15]. Let $Q=\left(q_{1}, q_{2}, \cdots, q_{n}\right) \in R^{n}$ be a parameter vector, and $\alpha_{i}(i=1,2, \cdots, n)$ be a collection of nonnegative real numbers. If

$$
M M^{Q}\left(\alpha_{1}, \alpha_{2}, \cdots, \alpha_{n}\right)=\left(\frac{1}{n !} \sum_{\theta \in S_{n}} \prod_{j=1}^{n} \alpha_{\theta(j)}^{q_{j}}\right)^{\sum_{j=1}^{\frac{1}{n} q_{j}}}
$$

where $M M^{Q}$ is called the Muirhead mean $(M M)$ operator and $\theta(j)(j=1,2, \cdots, n)$ is any a permutation of $(1,2, \cdots, n)$, and $S_{n}$ is the collection of all permutation of $(1,2, \cdots, n)$.

Definition 7. Let $Q=\left(q_{1}, q_{2}, \cdots, q_{n}\right) \in R^{n}$ be a parameter vector, and $\alpha_{i}=\left(\mu_{P_{i}}, v_{P_{i}}\right),(i=1,2, \cdots, n)$ be a collection of PFNs. If

$$
\operatorname{PFMM}^{Q}\left(\alpha_{1}, \alpha_{2}, \cdots, \alpha_{n}\right)=\left(\frac{1}{n !} \sum_{\theta \in S_{n}} \prod_{j=1}^{n} \alpha_{\theta(j)}^{q_{j}}\right)^{\frac{1}{\sum_{j=1}^{n} q_{j}}} .
$$

where PFMMQ is called the PFMM operator and $\theta(j)(j=1,2, \cdots, n)$ is any a permutation of $(1,2, \cdots, n)$, and $S_{n}$ is the collection of all permutation of $(1,2, \cdots, n)$.

Theorem 1. Let $\alpha_{i}=\left(\mu_{P_{i}}, v_{P_{i}}\right),(i=1,2, \cdots, n)$ be a collection of PFNs, then the aggregated value by using the PFMM operator is also a PFN, and

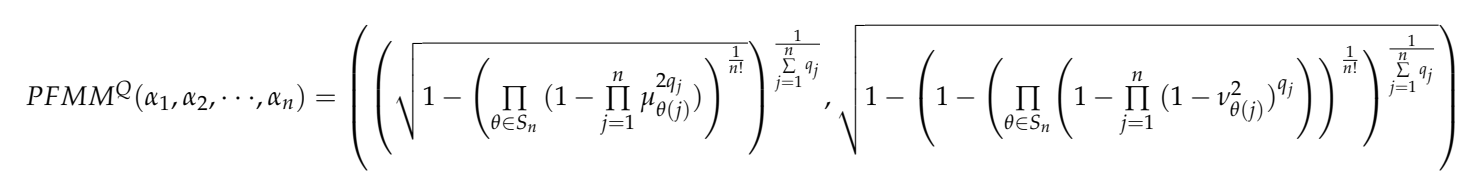

Proof. We need to prove that Equation (6) holds and is a PFN.

(1) Firstly, we prove that Equation (6) holds.

According to the operational laws (4) and (2) of Definition 2,

$$
\alpha_{\theta(j)}^{q_{j}}=\left(\mu_{\theta(j)^{\prime}}^{q_{j}} \sqrt{1-\left(1-v_{\theta(j)}^{2}\right)^{q_{j}}}\right), \text { and, } \prod_{j=1}^{n} \alpha_{\theta(j)}^{q_{j}}=\left(\prod_{j=1}^{n} \mu_{\theta(j)^{\prime}}^{q_{j}} \sqrt{1-\prod_{j=1}^{n}\left(1-v_{\theta(j)}^{2}\right)^{q_{j}}}\right)
$$

then

$$
\sum_{\theta \in S_{n}} \prod_{j=1}^{n} \alpha_{\theta(j)}^{q_{j}}=\left(\sqrt{1-\prod_{\theta \in S_{n}}\left(1-\prod_{j=1}^{n} \mu_{\theta(j)}^{2 q_{j}}\right)}, \prod_{\theta \in S_{n}} \sqrt{1-\prod_{j=1}^{n}\left(1-v_{\theta(j)}^{2}\right)^{q_{j}}}\right)
$$


further, based on operational law (3), we can get

$$
\frac{1}{n !} \sum_{\theta \in S_{n}} \prod_{j=1}^{n} \alpha_{\theta(j)}^{q_{j}}=\left(\sqrt{1-\left(\prod_{\theta \in S_{n}}\left(1-\prod_{j=1}^{n} \mu_{\theta(j)}^{2 q_{j}}\right)\right)^{\frac{1}{n !}}},\left(\prod_{\theta \in S_{n}} \sqrt{1-\prod_{j=1}^{n}\left(1-v_{\theta(j)}^{2}\right)^{q_{j}}}\right)^{\frac{1}{n !}}\right) .
$$

Consequently, we have

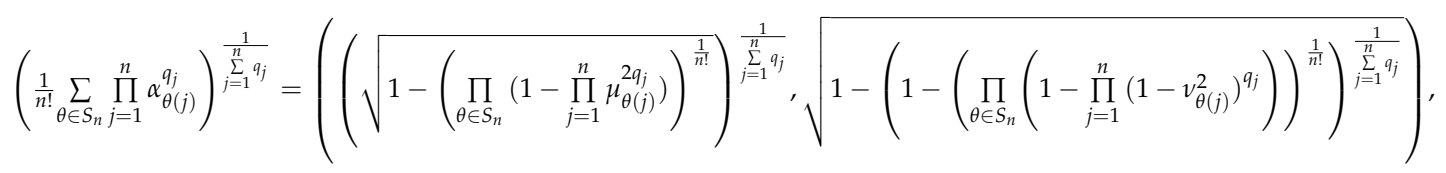

which illustrates that Equation (6) holds.

(2) In what follows, we will prove that Equation (6) is a PFN.

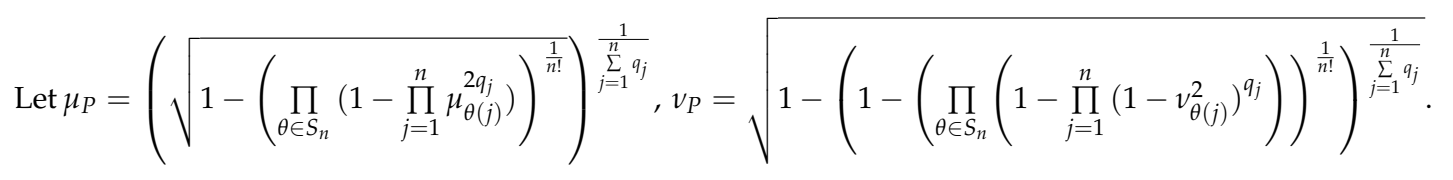

Then we need to prove that Equation (6) satisfies the following two conditions.
(a) $0 \leq \mu_{P} \leq 1$, and $0 \leq v_{P} \leq 1$;
(b) $\mu_{P}^{2}+v_{P}^{2} \leq 1$.
(a) According to Definition 1,

$$
\mu_{\theta(j)}^{2 q_{j}} \in[0,1] \text { and } \prod_{j=1}^{n} \mu_{\theta(j)}^{2 q_{j}} \in[0,1],
$$

then we have

$$
\prod_{\theta \in S_{n}}\left(1-\prod_{j=1}^{n} \mu_{\theta(j)}^{2 q_{j}}\right) \in[0,1],\left(\prod_{\theta \in S_{n}}\left(1-\prod_{j=1}^{n} \mu_{\theta(j)}^{2 q_{j}}\right)\right)^{\frac{1}{n !}} \in[0,1], \text { and } \sqrt{1-\left(\prod_{\theta \in S_{n}}\left(1-\prod_{j=1}^{n} \mu_{\theta(j)}^{2 q_{j}}\right)\right)^{\frac{1}{n !}}} \in[0,1],
$$

further

$$
\left(\sqrt{1-\left(\prod_{\theta \in S_{n}}\left(1-\prod_{j=1}^{n} \mu_{\theta(j)}^{2 q_{j}}\right)\right)^{\frac{1}{n !}}}\right)^{\frac{1}{\sum_{j=1}^{n} q_{j}}} \in[0,1] \text {, i.e., } 0 \leq \mu_{P} \leq 1 .
$$

Similarly, we can get $0 \leq v_{P} \leq 1$. So condition (a) is satisfied.

(b) Based on $\mu_{\theta(j)}^{2}+v_{\theta(j)}^{2} \leq 1$, then $\mu_{\theta(j)}^{2} \leq 1-v_{\theta(j)}^{2}$, we yield the inequality as follows:

$$
\begin{aligned}
& \mu_{P}^{2}+v_{P}^{2}=\left(1-\left(\prod_{\theta \in S_{n}}\left(1-\prod_{j=1}^{n} \mu_{\theta(j)}^{2 q_{j}}\right)\right)^{\frac{1}{n !}}\right)^{\frac{1}{\sum_{j=1}^{n} q_{j}}}+1-\left(1-\left(\prod_{\theta \in S_{n}}\left(1-\prod_{j=1}^{n}\left(1-v_{\theta(j)}^{2}\right)^{q_{j}}\right)\right)^{\frac{1}{n !}}\right)_{j=1}^{\frac{1}{\sum_{j}^{n} q_{j}}} \\
\leq & \left(1-\left(\prod_{\theta \in S_{n}}\left(1-\prod_{j=1}^{n}\left(1-v_{\theta(j)}^{2}\right)^{q_{j}}\right)\right)^{\frac{1}{n !}}\right)^{\frac{1}{\sum_{j=1}^{n} q_{j}}}+1-\left(1-\left(\prod_{\theta \in S_{n}}\left(1-\prod_{j=1}^{n}\left(1-v_{\theta(j)}^{2}\right)^{q_{j}}\right)\right)^{\frac{1}{n !}}\right)^{\frac{1}{\sum_{j=1}^{n} q_{j}}}=1,
\end{aligned}
$$

i.e., $\mu_{P}^{2}+v_{P}^{2} \leq 1$. Consequently, condition (b) is satisfied.

Based on the proof above, we know that theorem 1 holds.

In what follows, we will explore some properties of the PFMM operator. 
Property 1 (Idempotency). Let $\alpha_{i}=\left(\mu_{P_{i}}, v_{P_{i}}\right),(i=1,2, \cdots, n)$ are equal, i.e., $\alpha_{i}=\alpha=\left(\mu_{P}, v_{P}\right)$ for all $i$, then

$$
\operatorname{PFMM}^{Q}\left(\alpha_{1}, \alpha_{2}, \cdots, \alpha_{n}\right)=\alpha=\left(\mu_{P}, v_{P}\right)
$$

Proof. Since $\alpha_{i}=\alpha=\left(\mu_{P}, v_{P}\right)$, according to the Theorem 1 yields

$$
\begin{aligned}
& \operatorname{PFMM}^{Q}\left(\alpha_{1}, \alpha_{2}, \cdots, \alpha_{n}\right)=\left(\left(\sqrt{1-\left(\prod_{\theta \in S_{n}}\left(1-\prod_{j=1}^{n} \mu_{P}^{2 q_{j}}\right)\right)^{\frac{1}{n !}}}\right)^{\sum_{j=1}^{\frac{1}{n} q_{j}}}, \sqrt{1-\left(1-\left(\prod_{\theta \in S_{n}}\left(1-\prod_{j=1}^{n}\left(1-v_{P}^{2}\right)^{q_{j}}\right)\right)^{\frac{1}{n !}}\right)^{\frac{1}{\sum_{j=1}^{n} q_{j}}}}\right), \\
& =\left(\left(\sqrt{1-\left(\prod_{\theta \in S_{n}}\left(1-\mu_{P}^{2 \sum_{j=1}^{n} q_{j}}\right)\right)^{\frac{1}{n !}}}, \sqrt{1-\left(1-\left(\prod_{\theta \in S_{n}}\left(1-\left(1-v_{P}^{2}\right)^{\sum_{j=1}^{n} q_{j}}\right)\right)^{\frac{1}{\sum_{j=1} q_{j}}}\right)^{\frac{1}{n !}}}\right)\right. \\
& =\left(\sqrt{1-\left(\left(1-\mu_{P}^{2 \sum_{j=1}^{n} q_{j} n !}\right)\right)^{n !}}, \sqrt{\left.1-\left(1-\left(\left(1-\left(1-v_{P}^{2}\right)^{\sum_{j=1}^{n} q_{j}}\right)^{n !}\right)^{n !}\right)^{\frac{1}{n !}}\right)_{j=1}^{\frac{1}{n} q_{j}}}\right) \\
& =\left(\left(\sqrt{\mu_{P}^{2 \sum_{j=1}^{n} q_{j}}}\right)^{\frac{1}{\sum_{j=1}^{n} q_{j}}}, \sqrt{1-\left(\left(1-v_{P}^{2}\right)^{\sum_{j=1}^{n} q_{j}}\right)^{\sum_{j=1}^{n} q_{j}}}\right)=\left(\mu_{P}, v_{P}\right) .
\end{aligned}
$$

Property 2 (Monotonicity). Let $\alpha_{i}=\left(\mu_{P_{i}}, v_{P_{i}}\right)$ and $\hat{\alpha}_{i}=\left(\hat{\mu}_{P_{i}}, \hat{v}_{P_{i}}\right),(i=1,2, \cdots, n)$ be two collections of PFNs. Through using the PFMM operator, if $\mu_{P_{i}} \geq \hat{\mu}_{P_{i}}$ and $v_{P_{i}} \leq \hat{v}_{P_{i}}$,

$$
\operatorname{PFMM}^{Q}\left(\alpha_{1}, \alpha_{2}, \cdots, \alpha_{n}\right) \geq \operatorname{PFMM}^{Q}\left(\hat{\alpha}_{1}, \hat{\alpha}_{2}, \cdots, \hat{\alpha}_{n}\right) \text {. }
$$

Proof. Let

$$
\begin{aligned}
& =\left(\left(\sqrt{1-\left(\prod_{\theta \in S_{n}}\left(1-\prod_{j=1}^{n} \mu_{\theta(j)}^{2 q_{j}}\right)\right)^{\frac{1}{n !}}}\right)_{j=1}^{\sum_{j=1}^{n} q_{j}}, \sqrt{1-\left(1-\left(\prod_{\theta \in S_{n}}\left(1-\prod_{j=1}^{n}\left(1-v_{\theta(j)}^{2}\right)^{q_{j}}\right)\right)^{\frac{1}{n !}}\right)^{\frac{1}{\sum_{j=1}^{n} q_{j}}}}=\left(\alpha_{1}, \alpha_{2}, \cdots, \alpha_{n}\right)\right. \\
& =\left(\left(\sqrt{1-\left(\prod_{\theta \in S_{n}}\left(1-\prod_{j=1}^{n} \hat{\mu}_{\theta(j)}^{2 q_{j}}\right)\right)^{\frac{1}{n !}}}\right)^{\frac{1}{\sum_{j=1}^{n} q_{j}}}, \sqrt{1-\left(1-\left(\prod_{\theta \in S_{n}}\left(1-\prod_{j=1}^{n}\left(1-\hat{v}_{\theta(j)}^{2}\right)^{q_{j}}\right)\right)^{\frac{1}{n !}}\right)^{\frac{1}{\sum_{j=1}^{n} q_{j}}}}=\left(\hat{\mu}_{P}, \hat{v}_{P}\right) .\right.
\end{aligned}
$$

Since $\mu_{P_{i}} \geq \hat{\mu}_{P_{i}}$, based on the operational laws of Definition 2, we have

$$
\mu_{\theta(j)}^{2 q_{j}} \geq \hat{\mu}_{\theta(j)}^{2 q_{j}} \text { and } \prod_{j=1}^{n} \mu_{\theta(j)}^{2 q_{j}} \geq \prod_{j=1}^{n} \hat{\mu}_{\theta(j)}^{2 q_{j}} \text { then } \prod_{\theta \in S_{n}}\left(1-\prod_{j=1}^{n} \mu_{\theta(j)}^{2 q_{j}}\right) \leq \prod_{\theta \in S_{n}}\left(1-\prod_{j=1}^{n} \hat{\mu}_{\theta(j)}^{2 q_{j}}\right)
$$

and

$$
\left(\prod_{\theta \in S_{n}}\left(1-\prod_{j=1}^{n} \mu_{\theta(j)}^{2 q_{j}}\right)\right)^{\frac{1}{n !}} \leq\left(\prod_{\theta \in S_{n}}\left(1-\prod_{j=1}^{n} \hat{\mu}_{\theta(j)}^{2 q_{j}}\right)\right)^{\frac{1}{n !}}
$$


Further,

$1-\left(\prod_{\theta \in S_{n}}\left(1-\prod_{j=1}^{n} \mu_{\theta(j)}^{2 q_{j}}\right)\right)^{\frac{1}{n !}} \geq 1-\left(\prod_{\theta \in S_{n}}\left(1-\prod_{j=1}^{n} \hat{\mu}_{\theta(j)}^{2 q_{j}}\right)\right)^{\frac{1}{n !}}, \sqrt{1-\left(\prod_{\theta \in S_{n}}\left(1-\prod_{j=1}^{n} \mu_{\theta(j)}^{2 q_{j}}\right)\right)^{\frac{1}{n !}}} \geq \sqrt{1-\left(\prod_{\theta \in S_{n}}\left(1-\prod_{j=1}^{n} \hat{\mu}_{\theta(j)}^{2 q_{j}}\right)\right)^{\frac{1}{n !}}}$

and

$$
\left(\sqrt{1-\left(\prod_{\theta \in S_{n}}\left(1-\prod_{j=1}^{n} \mu_{\theta(j)}^{2 q_{j}}\right)\right)^{\frac{1}{n !}}}\right)^{\frac{1}{\sum_{j=1}^{n} q_{j}}} \geq\left(\sqrt{1-\left(\prod_{\theta \in S_{n}}\left(1-\prod_{j=1}^{n} \hat{\mu}_{\theta(j)}^{2 q_{j}}\right)\right)^{\frac{1}{n !}}}\right)^{\frac{1}{\sum_{j=1}^{n} q_{j}}} .
$$

i.e., $\mu_{P} \geq \hat{\mu}_{P}$. Similarly, we also yield $v_{P} \leq \hat{v}_{P}$.

Consequently, $\operatorname{PFMM}^{\mathrm{Q}}\left(\alpha_{1}, \alpha_{2}, \cdots, \alpha_{n}\right) \geq \operatorname{PFMM}^{\mathrm{Q}}\left(\hat{\alpha}_{1}, \hat{\alpha}_{2}, \cdots, \hat{\alpha}_{n}\right)$ holds.

Property 3 (Boundedness). Let $\alpha_{i}=\left(\mu_{P_{i}}, v_{P_{i}}\right),(i=1,2, \cdots, n)$ be a collections of PFNs, $\alpha^{+}=$ $\left(\max \left(\mu_{P_{i}}\right), \min \left(\nu_{P_{i}}\right)\right)$ and $\alpha^{-}=\left(\min \left(\mu_{P_{i}}\right), \max \left(\nu_{P_{i}}\right)\right)$, then

$$
\alpha^{+} \geq \operatorname{PFMM}^{Q}\left(\alpha_{1}, \alpha_{2}, \cdots, \alpha_{n}\right) \geq \alpha^{-} .
$$

Proof. Based on Properties 1 and 2,

$\operatorname{PFMM}^{Q}\left(\alpha_{1}, \alpha_{2}, \cdots, \alpha_{n}\right) \leq \operatorname{PFMM}^{Q}\left(\alpha^{+}, \alpha^{+}, \cdots, \alpha^{+}\right)=\alpha^{+}$,

$\operatorname{PFMM}^{Q}\left(\alpha_{1}, \alpha_{2}, \cdots, \alpha_{n}\right) \geq \operatorname{PFMM}^{Q}\left(\alpha^{-}, \alpha^{-}, \cdots, \alpha^{-}\right)=\alpha^{-}$.

So, we can get $\alpha^{+} \geq \operatorname{PFMM} M^{Q}\left(\alpha_{1}, \alpha_{2}, \cdots, \alpha_{n}\right) \geq \alpha^{-}$.

In what follows, we will discuss some special cases of the PFMM operator through changing the values of parameter vector $Q$.

(1) When $Q=(1,0, \cdots, 0)$, Equation (6) is transformed into a Pythagorean fuzzy arithmetic averaging operator.

$$
\operatorname{PFMM}^{(1,0, \cdots, 0)}\left(\alpha_{1}, \alpha_{2}, \cdots, \alpha_{n}\right)=\frac{1}{n} \sum_{i=1}^{n} \alpha_{i}=\left(\sqrt{1-\prod_{i=1}^{n}\left(1-\mu_{P_{i}}^{2}\right)^{\frac{1}{n}}}, \prod_{i=1}^{n}\left(v_{P_{i}}\right)^{\frac{1}{n}}\right) .
$$

(2) When $Q=(\lambda, 0, \cdots, 0)$, Equation (6) is transformed into a Pythagorean fuzzy generalized arithmetic averaging operator:

$$
\operatorname{PFMM}^{(\lambda, 0, \cdots, 0)}\left(\alpha_{1}, \alpha_{2}, \cdots, \alpha_{n}\right)=\left(\left(\sqrt{1-\prod_{i=1}^{n}\left(1-\mu_{P_{i}}^{2 \lambda}\right)^{\frac{1}{n}}}\right)^{\frac{1}{\lambda}}, \sqrt{1-\left(1-\prod_{i=1}^{n}\left(1-\left(1-v_{P_{i}}^{2}\right)^{\lambda}\right)^{\frac{1}{n}}\right)^{\frac{1}{\lambda}}}\right) .
$$

(3) When $Q=(1,1,0,0 \cdots, 0)$, Equation (6) is transformed into a Pythagorean fuzzy BM operator:

$$
\left.\operatorname{PFMM}^{(1,1,0,0 \cdots, 0)}\left(\alpha_{1}, \alpha_{2}, \cdots, \alpha_{n}\right)=\left(\sqrt{1-\left(\prod_{\substack{i, j=1 \\ i \neq j}}^{n}\left(1-\mu_{P_{i}}^{2} \mu_{P_{j}}^{2}\right)\right)^{\frac{1}{n(n-1)}}}\right)^{\frac{1}{2}}, \sqrt{1-\left(1-\prod_{\substack{i, j=1 \\ i \neq j}}^{n}\left(1-\left(1-v_{P_{i}}^{2}\right)\left(1-v_{P_{j}}^{2}\right)\right)^{\frac{1}{n(n-1)}}\right)^{\frac{1}{2}}}\right) .
$$


(4) When $Q=(\underbrace{1,1, \cdots, 1}_{k}, \underbrace{0,0, \cdots, 0}_{n-k})$, Equation (6) is transformed into a Pythagorean fuzzy MSM operator [25]:

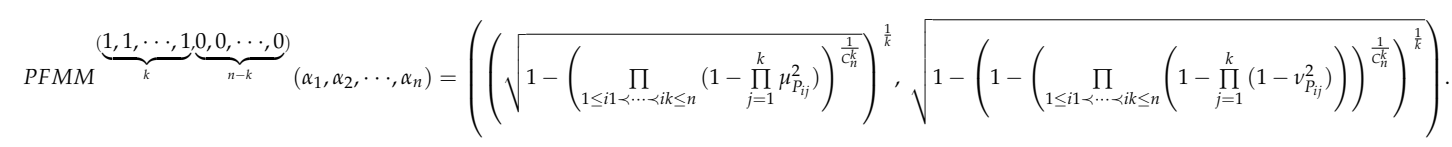

(5) When $Q=(1,1, \cdots, 1)$, Equation (6) is transformed into a Pythagorean fuzzy geometric averaging operator:

$$
\operatorname{PFMM}^{(1,1, \cdots, 1)}\left(\alpha_{1}, \alpha_{2}, \cdots, \alpha_{n}\right)=\left(\prod_{i=1}^{n} \alpha_{i}\right)^{\frac{1}{n}}=\left(\left(\prod_{i=1}^{n} \mu_{P_{i}}\right)^{\frac{1}{n}}, \sqrt{1-\prod_{i=1}^{n}\left(1-v_{P_{i}}^{2}\right)^{\frac{1}{n}}}\right) .
$$

(6) When $Q=(1 / n, 1 / n, \cdots, 1 / n)$, Equation (6) is transformed into a Pythagorean fuzzy geometric averaging operator:

$$
\operatorname{PFMM}^{(1 / n, 1 / n, \cdots, 1 / n)}\left(\alpha_{1}, \alpha_{2}, \cdots, \alpha_{n}\right)=\prod_{i=1}^{n} \alpha_{i}^{\frac{1}{n}}=\left(\left(\prod_{i=1}^{n} \mu_{P_{i}}\right)^{\frac{1}{n}}, \sqrt{1-\prod_{i=1}^{n}\left(1-v_{P_{i}}^{2}\right)^{\frac{1}{n}}}\right) .
$$

\subsection{The PFWMM Operator}

Definition 8. Let $Q=\left(q_{1}, q_{2}, \cdots, q_{n}\right) \in R^{n}$ be a parameter vector, $\alpha_{i}=\left(\mu_{P_{i}}, v_{P_{i}}\right),(i=1,2, \cdots, n)$ be a collection of PFNs, and $w=\left(w_{1}, w_{2}, \cdots, w_{n}\right)^{T}$ be the weight vector of $\alpha_{i}$, where $w_{i}$ indicates the importance degree of $\alpha_{i}$, satisfying $w_{i} \in[0,1]$ and $\sum_{i=1}^{n} w_{i}=1$. If

$$
\operatorname{PFWMM}^{Q}\left(\alpha_{1}, \alpha_{2}, \cdots, \alpha_{n}\right)=\left(\frac{1}{n !} \sum_{\theta \in S_{n}} \prod_{j=1}^{n}\left(n w_{\theta(j)} \alpha_{\theta(j)}\right)^{q_{j}}\right)^{\frac{1}{\sum_{j=1}^{n} q_{j}}},
$$

where PFWMM ${ }^{Q}$ is called the PFWMM operator and $\theta(j)(j=1,2, \cdots, n)$ is any a permutation of $(1,2, \cdots, n)$, and $S_{n}$ is the collection of all permutation of $(1,2, \cdots, n)$.

Theorem 2. Let $Q=\left(q_{1}, q_{2}, \cdots, q_{n}\right) \in R^{n}$ be a parameter vector, $\alpha_{i}=\left(\mu_{P_{i}}, v_{P_{i}}\right),(i=1,2, \cdots, n)$ be a collection of PFNs, and $w=\left(w_{1}, w_{2}, \cdots, w_{n}\right)^{T}$ be the weight vector of $\alpha_{i}$, where $w_{i}$ indicates the importance degree of $\alpha_{i}$, satisfying $w_{i} \in[0,1]$ and $\sum_{i=1}^{n} w_{i}=1$. Then, the aggregated value by using the PFWMM operator is also a PFN, and

$$
=\left(\left(\sqrt{\left.1-\left(\prod_{\theta \in S_{n}}\left(1-\prod_{j=1}^{n}\left(1-\left(1-\mu_{\theta(j)}^{2}\right)^{n w_{\theta(j)}}\right)^{q_{j}}\right)\right)^{\frac{1}{n}}\right)^{\sum_{j=1}^{n} q_{j}}, \sqrt{1-\left(1-\left(\prod_{\theta \in S_{n}}\left(1-\prod_{j=1}^{n}\left(1-v_{\theta(j)}^{2 n w_{\theta(j)}}\right)^{q_{j}}\right)\right)^{\frac{1}{n}}\right)^{\frac{1}{\sum_{j=1}^{n} q_{j}}}} .} .\right.\right.
$$

Proof. Based on the operational law (3) in Definition 2, $n w_{\theta(j)} \alpha_{\theta(j)}=\left(\sqrt{1-\left(1-\mu_{\theta(j)}^{2}\right)^{n z w_{\theta(j)}}}, v_{\theta(j)}^{n w_{\theta(j)}}\right)$, and we can replace $\mu_{\theta(j)}$ and $v_{\theta(j)}$ with $\sqrt{1-\left(1-\mu_{\theta(j)}^{2}\right)^{n w_{\theta(j)}}}$ and $v_{\theta(j)}^{n w_{\theta(j)}}$, respectively, in Equation (6), thus obtaining Equation (14). Since $\alpha_{\theta(j)}$ is a PFN, then $n w_{\theta(j)} \alpha_{\theta(j)}$ is also a PFN. Similar to the proof of Theorem 1, we know Equation (14) is also a PFN. 
In the following, we will discuss some desirable properties of the PFWMM operator.

Property 4 (Monotonicity). Let $\alpha_{i}=\left(\mu_{P_{i}}, v_{P_{i}}\right)$ and $\hat{\alpha}_{i}=\left(\hat{\mu}_{P_{i}}, \hat{v}_{P_{i}}\right),(i=1,2, \cdots, n)$ be two collections of PFNs. Through using the PFMM operator, if $\mu_{P_{i}} \geq \hat{\mu}_{P_{i}}$ and $v_{P_{i}} \leq \hat{v}_{P_{i}}$, then

$$
\operatorname{PFWMM}^{\mathrm{Q}}\left(\alpha_{1}, \alpha_{2}, \cdots, \alpha_{n}\right) \geq \operatorname{PFWMM}^{\mathrm{Q}}\left(\hat{\alpha}_{1}, \hat{\alpha}_{2}, \cdots, \hat{\alpha}_{n}\right) .
$$

The Proof of Property 4 is similar to that of Property 2, so is omitted here.

Property 5 (Boundedness). Let $\alpha_{i}=\left(\mu_{P_{i}}, v_{P_{i}}\right),(i=1,2, \cdots, n)$ be a collections of PFNs, $\alpha^{+}=$ $\left(\max \left(\mu_{P_{i}}\right), \min \left(v_{P_{i}}\right)\right)$ and $\alpha^{-}=\left(\min \left(\mu_{P_{i}}\right), \max \left(v_{P_{i}}\right)\right)$, then

$$
\alpha^{+} \geq \operatorname{PFWMM}^{Q}\left(\alpha_{1}, \alpha_{2}, \cdots, \alpha_{n}\right) \geq \alpha^{-} .
$$

The Proof of Property 5 is similar to that of Property 3, so is omitted here.

Theorem 3. The PFMM operator is a special case of the PFWMM operator.

Proof. When $w=(1 / n, 1 / n, \cdots, 1 / n)^{T}$

$$
\begin{aligned}
& =\left(\left(\sqrt{\left.\left.1-\left(\prod_{\theta \in S_{n}}\left(1-\prod_{j=1}^{n}\left(1-\left(1-\mu_{\theta(j)}^{2}\right)^{n \times \frac{1}{n}}\right)^{q_{j}}\right)\right)^{\frac{1}{n}}\right)^{\sum_{j=1}^{n} q_{j}}, \sqrt{1-\left(1-\left(\prod_{\theta \in S_{n}}\left(1-\prod_{j=1}^{n}\left(1-v_{\theta(j)}^{2 n \times \frac{1}{n}}\right)^{q_{j}}\right)\right)^{\frac{1}{n !}}\right)^{\frac{1}{\sum_{j=1}^{n} q_{j}}}}\right)}\right.\right.
\end{aligned}
$$

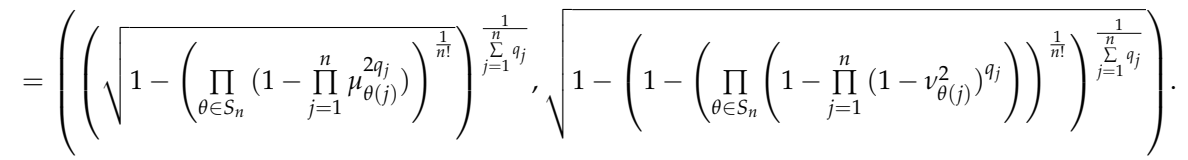

Theorem 4. The Pythagorean fuzzy weighted averaging operator [6] is a special case of the PFWMM operator.

Proof. When $Q=(1,0, \cdots, 0)$,

$$
\begin{aligned}
& =\left(\left(\sqrt{\left.\left.1-\left(\prod_{\theta \in S_{n}}\left(1-\prod_{j=1}^{n}\left(1-\left(1-\mu_{\theta(j)}^{2}\right)^{n w_{\theta(j)}}\right)^{q_{j}}\right)\right)^{\frac{1}{n !}}\right)^{\frac{1}{\sum_{j=1}^{n} q_{j}}}, \sqrt{1-\left(1-\left(\prod_{\theta \in S_{n}}\left(1-\prod_{j=1}^{n}\left(1-v_{\theta(j)}^{2 n w_{\theta(j)}}\right)^{q_{j}}\right)\right)^{\frac{1}{n !}}\right)^{\frac{1}{\sum_{j=1}^{n} q_{j}}}}\right)}\right.\right. \\
& =\left(\sqrt{1-\left(\prod_{j=1}^{n}\left(1-\mu \mu_{P_{j}}^{2}\right)^{n w w_{j}}\right)^{\frac{1}{n}}}, \sqrt{1-\left(1-\left(\prod_{j=1}^{n} v_{P_{j}}^{2 n w w_{j}}\right)^{\frac{1}{n}}\right)}\right) \\
& =\left(\sqrt{1-\prod_{j=1}^{n}\left(1-\mu_{P_{j}}^{2}\right)^{w_{j}}}, \prod_{j=1}^{n} v_{P_{j}}^{w_{j}}\right) .
\end{aligned}
$$

\section{Some Pythagorean Fuzzy Dual MM Operators}

In this section, we will define the Pythagorean fuzzy dual MM (PFDMM) operator and Pythagorean fuzzy dual weighted MM (PFDWMM) operator. 


\subsection{The PFDMM Operator}

Definition 9. Let $Q=\left(q_{1}, q_{2}, \cdots, q_{n}\right) \in R^{n}$ be a parameter vector, and $\alpha_{i}=\left(\mu_{P_{i}}, v_{P_{i}}\right),(i=1,2, \cdots, n)$ be a collection of PFNs. If

$$
\operatorname{PFDMM}^{Q}\left(\alpha_{1}, \alpha_{2}, \cdots, \alpha_{n}\right)=\frac{1}{\sum_{j=1}^{n} q_{j}}\left(\prod_{\theta \in S_{n}} \sum_{j=1}^{n}\left(q_{j} \alpha_{\theta(j)}\right)\right)^{\frac{1}{n !}},
$$

where PFDMM ${ }^{Q}$ is called the PFDMM operator and $\theta(j)(j=1,2, \cdots, n)$ is any a permutation of $(1,2, \cdots, n)$, and $S_{n}$ is the collection of all permutations of $(1,2, \cdots, n)$.

Theorem 5. Let $\alpha_{i}=\left(\mu_{P_{i}}, v_{P_{i}}\right),(i=1,2, \cdots, n)$ be a collection of PFNs, then the aggregated value by using the PFDMM operator is also a PFN, and

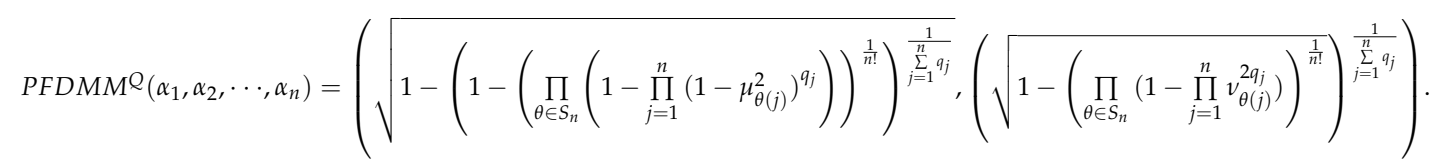

Proof. We need to prove that Equation (16) holds and is a PFN.

(1) Firstly, we will prove that Equation (16) holds.

According to laws (3) and (1) in Definition 2,

$$
q_{j} \alpha_{\theta(j)}=\left(\sqrt{1-\left(1-\mu_{\theta(j)}^{2}\right)^{q_{j}}}, v_{\theta(j)}^{q_{j}}\right) \text { and } \sum_{j=1}^{n}\left(q_{j} \alpha_{\theta(j)}\right)=\left(\sqrt{1-\prod_{j=1}^{n}\left(1-\mu_{\theta(j)}^{2}\right)^{q_{j}}}, \prod_{j=1}^{n} v_{\theta(j)}^{q_{j}}\right) \text {. }
$$

then, based on laws (1) and (3) in Definition 2, we can obtain

$$
\prod_{\theta \in S_{n}} \sum_{j=1}^{n}\left(q_{j} \alpha_{\theta(j)}\right)=\left(\prod_{\theta \in S_{n}} \sqrt{1-\prod_{j=1}^{n}\left(1-\mu_{\theta(j)}^{2}\right)^{q_{j}}}, \sqrt{1-\prod_{\theta \in S_{n}}\left(1-\prod_{j=1}^{n} v_{\theta(j)}^{2 q_{j}}\right)}\right)
$$

and

$$
\left(\prod_{\theta \in S_{n}} \sum_{j=1}^{n}\left(q_{j} \alpha_{\theta(j)}\right)\right)^{\frac{1}{n !}}=\left(\left(\prod_{\theta \in S_{n}} \sqrt{1-\prod_{j=1}^{n}\left(1-\mu_{\theta(j)}^{2}\right)^{q_{j}}}\right)^{\frac{1}{n !}}, \sqrt{1-\left(\prod_{\theta \in S_{n}}\left(1-\prod_{j=1}^{n} v_{\theta(j)}^{2 q_{j}}\right)\right)^{\frac{1}{n !}}} .\right.
$$

Further,

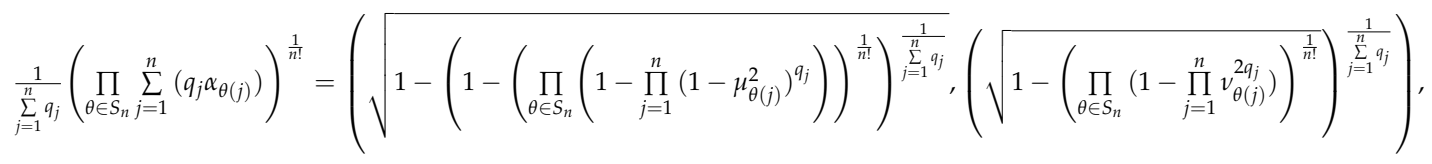

which illustrates that Equation (16) holds.

(2) In the following, we will prove that Equation (16) is a PFN.

$$
\text { Let } \mu_{P}=\sqrt{1-\left(1-\left(\prod_{\theta \in S_{n}}\left(1-\prod_{j=1}^{n}\left(1-\mu_{\theta(j)}^{2}\right)^{q_{j}}\right)\right)^{\left.\frac{1}{n !}\right)^{\frac{1}{n} \sum_{j=1}^{n} q_{j}}}\right.}
$$


$v_{P}=\left(\sqrt{1-\left(\prod_{\theta \in S_{n}}\left(1-\prod_{j=1}^{n} v_{\theta(j)}^{2 q_{j}}\right)\right)^{\frac{1}{n !}}}\right)^{\sum_{j=1}^{n} q_{j}}$

Then we also need to prove that Equation (16) satisfies the following two conditions.

(a) $0 \leq \mu_{P} \leq 1$, and $0 \leq v_{P} \leq 1$;

(b) $\mu_{P}^{2}+v_{P}^{2} \leq 1$.

(a) Based on Definition 1,

$$
1-\mu_{\theta(j)}^{2} \in[0,1] \text { and }\left(1-\mu_{\theta(j)}^{2}\right)^{q_{j}} \in[0,1]
$$

we get

$$
\prod_{j=1}^{n}\left(1-\mu_{\theta(j)}^{2}\right)^{q_{j}} \in[0,1] \text { and } \prod_{\theta \in S_{n}}\left(1-\prod_{j=1}^{n}\left(1-\mu_{\theta(j)}^{2}\right)^{q_{j}}\right) \in[0,1]
$$

Further,

$$
\left(\prod_{\theta \in S_{n}}\left(1-\prod_{j=1}^{n}\left(1-\mu_{\theta(j)}^{2}\right)^{q_{j}}\right)\right)^{\frac{1}{n !}} \in[0,1] \text { and }\left(1-\left(\prod_{\theta \in S_{n}}\left(1-\prod_{j=1}^{n}\left(1-\mu_{\theta(j)}^{2}\right)^{q_{j}}\right)\right)^{\frac{1}{n !}}\right)^{\sum_{j=1}^{\frac{1}{n} q_{j}}} \in[0,1]
$$

then

$$
\sqrt{1-\left(1-\left(\prod_{\theta \in S_{n}}\left(1-\prod_{j=1}^{n}\left(1-\mu_{\theta(j)}^{2}\right)^{q_{j}}\right)\right)^{\left.\frac{1}{n !}\right)^{\frac{1}{\sum_{j=1}^{n} q_{j}}}}\right.} \in[0,1]
$$

i.e., $0 \leq \mu_{P} \leq 1$. Similarly, we can yield $0 \leq v_{P} \leq 1$. Therefore, condition (a) is satisfied.

(b) Because $\mu_{\theta(j)}^{2}+v_{\theta(j)}^{2} \leq 1$, then $v_{\theta(j)}^{2} \leq 1-\mu_{\theta(j)}^{2}$, we can obtain the inequality as follows:

$$
\begin{aligned}
& \mu_{P_{i}}^{2}+v_{P_{i}}^{2}=1-\left(1-\left(\prod_{\theta \in S_{n}}\left(1-\prod_{j=1}^{n}\left(1-\mu_{\theta(j)}^{2}\right)^{q_{j}}\right)\right)^{\frac{1}{n !}}\right)^{\frac{1}{\sum_{j=1}^{n} q_{j}}}+\left(1-\left(\prod_{\theta \in S_{n}}\left(1-\prod_{j=1}^{n} v_{\theta(j)}^{2 q_{j}}\right)\right)^{\frac{1}{n !}}\right)^{\frac{1}{\sum_{j=1}^{n} q_{j}}} \\
\leq & 1-\left(1-\left(\prod_{\theta \in S_{n}}\left(1-\prod_{j=1}^{n}\left(1-\mu_{\theta(j)}^{2}\right)^{q_{j}}\right)\right)^{\frac{1}{n !}}\right)^{\frac{1}{\sum_{j=1}^{n} q_{j}}}+\left(1-\left(\prod_{\theta \in S_{n}}\left(1-\prod_{j=1}^{n}\left(1-\mu_{\theta(j)}^{2}\right)^{q_{j}}\right)\right)^{\frac{1}{n !}}\right)^{\frac{1}{\sum_{j=1}^{n} q_{j}}}=1,
\end{aligned}
$$

i.e., $\mu_{P}^{2}+v_{P}^{2} \leq 1$. Consequently, condition (b) is satisfied.

Based on the proof above, we know that Theorem 5 holds.

Similar to the properties of the PFMM operator, we can easily obtain some properties of the PFDMM operator as follows.

Property 6 (Idempotency). Let $\alpha_{i}=\left(\mu_{P_{i}}, v_{P_{i}}\right),(i=1,2, \cdots, n)$ are equal, i.e., $\alpha_{i}=\alpha=\left(\mu_{P}, v_{P}\right)$ for all $i$, then

$$
\operatorname{PFDMM}^{Q}\left(\alpha_{1}, \alpha_{2}, \cdots, \alpha_{n}\right)=\alpha=\left(\mu_{P}, v_{P}\right) .
$$

The Proof of Property 6 is similar to that of Property 1, so is omitted here.

Property 7 (Monotonicity). Let $\alpha_{i}=\left(\mu_{P_{i}}, v_{P_{i}}\right)$ and $\hat{\alpha}_{i}=\left(\hat{\mu}_{P_{i}}, \hat{v}_{P_{i}}\right),(i=1,2, \cdots, n)$ be two collections of PFNs. If $\mu_{P_{i}} \geq \hat{\mu}_{P_{i}}$ and $v_{P_{i}} \leq \hat{v}_{P_{i}}$ for all $i$, then

$$
\operatorname{PFDMM}^{Q}\left(\alpha_{1}, \alpha_{2}, \cdots, \alpha_{n}\right) \geq \operatorname{PFDMM}^{Q}\left(\hat{\alpha}_{1}, \hat{\alpha}_{2}, \cdots, \hat{\alpha}_{n}\right) .
$$


The Proof of Property 7 is similar to that of Property 2, so is omitted here.

Property 8 (Boundedness). Let $\alpha_{i}=\left(\mu_{P_{i}}, v_{P_{i}}\right),(i=1,2, \cdots, n)$ be a collections of PFNs, $\alpha^{+}=$ $\left(\max \left(\mu_{P_{i}}\right), \min \left(v_{P_{i}}\right)\right)$ and $\alpha^{-}=\left(\min \left(\mu_{P_{i}}\right), \max \left(v_{P_{i}}\right)\right)$, then

$$
\alpha^{+} \geq \operatorname{PFDMM}^{Q}\left(\alpha_{1}, \alpha_{2}, \cdots, \alpha_{n}\right) \geq \alpha^{-} .
$$

The Proof of Property 8 is similar to that of Property 3, so is omitted here.

In what follows, we will discuss some special cases of the PFDMM operator through changing the values of parameter vector $Q$.

(1). When $Q=(1,0, \cdots, 0)$, Equation (16) is transformed into a Pythagorean fuzzy geometric averaging operator:

$$
\operatorname{PFDMM}^{(1,0, \cdots, 0)}\left(\alpha_{1}, \alpha_{2}, \cdots, \alpha_{n}\right)=\left(\prod_{i=1}^{n}\left(\mu_{P_{i}}\right)^{\frac{1}{n}}, \sqrt{1-\prod_{i=1}^{n}\left(1-v_{P_{i}}^{2}\right)^{\frac{1}{n}}}\right) .
$$

(2). When $Q=(\lambda, 0, \cdots, 0)$, Equation (16) is transformed into a Pythagorean fuzzy generalized geometric averaging operator:

$$
\operatorname{PFDMM}^{(\lambda, 0, \cdots, 0)}\left(\alpha_{1}, \alpha_{2}, \cdots, \alpha_{n}\right)=\left(\sqrt{1-\left(1-\prod_{i=1}^{n}\left(1-\left(1-\mu_{P_{i}}^{2}\right)^{\lambda}\right)^{\frac{1}{n}}\right)^{\frac{1}{\lambda}}},\left(\sqrt{1-\prod_{i=1}^{n}\left(1-v_{P_{i}}^{2 \lambda}\right)^{\frac{1}{n}}}\right)^{\frac{1}{\lambda}}\right) .
$$

(3). When $Q=(1,1,0,0 \cdots, 0)$, Equation (16) is transformed into a Pythagorean fuzzy geometric BM operator:

$$
\operatorname{PFDMM}^{(1,1,0,0, \cdots, 0,0)}\left(\alpha_{1}, \alpha_{2}, \cdots, \alpha_{n}\right)=\left(\sqrt{1-\left(1-\prod_{\substack{i, j=1 \\ i \neq j}}^{n}\left(1-\left(1-\mu_{P_{i}}^{2}\right)\left(1-\mu_{P_{j}}^{2}\right)\right)^{\frac{1}{\pi(n-1)}}\right)^{\frac{1}{2}}},\left(\sqrt{1-\left(\prod_{\substack{i, j=1 \\ i \neq j}}^{n}\left(1-v_{P_{i}}^{2} v_{P_{j}}^{2}\right)\right)^{\frac{1}{\pi(n-1)}}}\right)^{\frac{1}{2}}\right) .
$$

(4). When $Q=(\underbrace{1,1, \cdots, 1}_{k}, \underbrace{0,0, \cdots, 0}_{n-k})$, Equation (16) is transformed into a Pythagorean fuzzy geometric MSM operator:

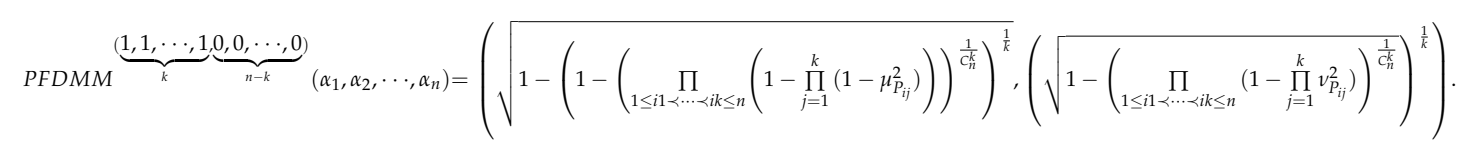

(5). When $Q=(1,1, \cdots, 1)$, Equation (16) is transformed into a Pythagorean fuzzy arithmetic averaging operator:

$$
\operatorname{PFDMM}^{(1,1, \cdots, 1)}\left(\alpha_{1}, \alpha_{2}, \cdots, \alpha_{n}\right)=\left(\sqrt{1-\prod_{i=1}^{n}\left(1-\mu_{P_{i}}^{2}\right)^{\frac{1}{n}}},\left(\prod_{i=1}^{n} v_{P_{i}}\right)^{\frac{1}{n}}\right) .
$$

(6). When $Q=(1 / n, 1 / n, \cdots, 1 / n)$, Equation (16) is transformed into a Pythagorean fuzzy arithmetic averaging operator:

$$
\operatorname{PFDMM}^{(1 / n, 1 / n, \cdots, 1 / n)}\left(\alpha_{1}, \alpha_{2}, \cdots, \alpha_{n}\right)=\left(\sqrt{1-\prod_{i=1}^{n}\left(1-\mu_{P_{i}}^{2}\right)^{\frac{1}{n}}},\left(\prod_{i=1}^{n} v_{P_{i}}\right)^{\frac{1}{n}}\right) .
$$




\subsection{The PFDWMM Operator}

Definition 10. Let $Q=\left(q_{1}, q_{2}, \cdots, q_{n}\right) \in R^{n}$ is parameter vector, $\alpha_{i}=\left(\mu_{P_{i}}, v_{P_{i}}\right),(i=1,2, \cdots, n)$ be a collection of PFNs, and $w=\left(w_{1}, w_{2}, \cdots, w_{n}\right)^{T}$ be the weighted vector of $\alpha_{i}$, where $w_{i}$ indicates the importance degree of $\alpha_{i}$, satisfying $w_{i} \in[0,1]$ and $\sum_{i=1}^{n} w_{i}=1$, If

$$
\operatorname{PFDWMM}^{Q}\left(\alpha_{1}, \alpha_{2}, \cdots, \alpha_{n}\right)=\frac{1}{\sum_{j=1}^{n} q_{j}}\left(\prod_{\theta \in S_{n}} \sum_{j=1}^{n}\left(q_{j} \alpha_{\theta(j)}^{n w_{\theta(j)}}\right)\right)^{\frac{1}{n !}} .
$$

then PFDWMMQ is called the PFDWMM operator.

Theorem 6. Let $Q=\left(q_{1}, q_{2}, \cdots, q_{n}\right) \in R^{n}$ be a parameter vector, $\alpha_{i}=\left(\mu_{P_{i}}, v_{P_{i}}\right),(i=1,2, \cdots, n)$ be a collection of PFNs, and $w=\left(w_{1}, w_{2}, \cdots, w_{n}\right)^{T}$ be the weight vector of $\alpha_{i}$, where $w_{i}$ indicates the importance degree of $\alpha_{i}$, satisfying $w_{i} \in[0,1]$ and $\sum_{i=1}^{n} w_{i}=1$. Then, the aggregated value by using the PFDWMM operator is also a PFN, and

$$
=\left(\sqrt{1-\left(1-\left(\prod_{\theta \in S_{n}}\left(1-\prod_{j=1}^{n}\left(1-\mu_{\theta(j)}^{2 n w_{\theta j}}\right)^{q_{j}}\right)\right)^{\frac{1}{n !}}\right)^{\sum_{j=1}^{n} q_{j}}}\left(\sqrt{1-\left(\prod_{\theta \in S_{n}}\left(1-\prod_{j=1}^{n}\left(1-\left(1-v_{\theta(j)}^{2}\right)^{n w w_{\theta(j)}}\right)^{q_{j}}\right)\right)^{\frac{1}{n !}}}\right)^{\frac{1}{\sum_{j=1}^{n} q_{j}}}\right) .
$$

Proof. According to operational law (4) in Definition 2, then $\alpha_{\theta(j)}^{n w w_{\theta(j)}}=\left(\mu_{\theta(j)}^{n w_{\theta(j)}}, \sqrt{1-\left(1-v_{\theta(j)}^{2}\right)^{n n w_{\theta(j)}}}\right)$, and we can replace $\mu_{\theta(j)}$ and $v_{\theta(j)}$ with $\mu_{\theta(j)}^{n w_{\theta(j)}}$ and $\sqrt{1-\left(1-v_{\theta(j)}^{2}\right)^{n w w_{\theta(j)}}}$, respectively, in Equation (16), to obtain Equation (24). Since $\alpha_{\theta(j)}$ is a PFN, $\alpha_{\theta(j)}^{n w_{\theta(j)}}$ is also a PFN. Similar to the proof of Theorem 5, we know Equation (24) is also a PFN.

In the following, we will discuss some desirable properties of the PFDWMM operator.

Property 9 (Monotonicicty). Let $\alpha_{i}=\left(\mu_{P_{i}}, v_{P_{i}}\right)$ and $\hat{\alpha}_{i}=\left(\hat{\mu}_{P_{i}}, \hat{v}_{P_{i}}\right),(i=1,2, \cdots, n)$ be two collections of PFNs. Through using the PFDWMM operator, if $\mu_{P_{i}} \geq \hat{\mu}_{P_{i}}$ and $v_{P_{i}} \leq \hat{v}_{P_{i}}$, then

$$
\operatorname{PFDWMM}^{\mathrm{Q}}\left(\alpha_{1}, \alpha_{2}, \cdots, \alpha_{n}\right) \geq \operatorname{PFDWMM}^{\mathrm{Q}}\left(\hat{\alpha}_{1}, \hat{\alpha}_{2}, \cdots, \hat{\alpha}_{n}\right) .
$$

The Proof of Property 9 is similar to that of Property 2, so is omitted here.

Property 10 (Boundedness). Let $\alpha_{i}=\left(\mu_{P_{i}}, v_{P_{i}}\right),(i=1,2, \cdots, n)$ be a collections of PFNs, $\alpha^{+}=$ $\left(\max \left(\mu_{P_{i}}\right), \min \left(v_{P_{i}}\right)\right)$ and $\alpha^{-}=\left(\min \left(\mu_{P_{i}}\right), \max \left(v_{P_{i}}\right)\right)$, then

$$
\alpha^{+} \geq \operatorname{PFDWMM}^{Q}\left(\alpha_{1}, \alpha_{2}, \cdots, \alpha_{n}\right) \geq \alpha^{-} .
$$

The Proof Property 10 is similar to that of Property 3, so is omitted here.

Theorem 7. The PFDMM operator is a special case of the PFDWMM operator. 
Proof. When $w=(1 / n, 1 / n, \cdots, 1 / n)^{T}$,

$$
\begin{aligned}
& =\left(\sqrt{1-\left(1-\left(\prod_{\theta \in S_{n}}\left(1-\prod_{j=1}^{n}\left(1-\mu_{\theta(j)}^{2 n \times \frac{1}{n}}\right)^{q_{j}}\right)\right)^{\frac{1}{n}}\right)^{\sum_{j=1}^{\frac{1}{n} q_{j}}},\left(\sqrt{\left.1-\left(\prod_{\theta \in S_{n}}\left(1-\prod_{j=1}^{n}\left(1-\left(1-v_{\theta(j)}^{2}\right)^{n \times \frac{1}{n}}\right)^{q_{j}}\right)\right)^{\frac{1}{n !}}\right)^{\frac{1}{\sum_{j=1}^{n} q_{j}}}}\right)}\right.
\end{aligned}
$$

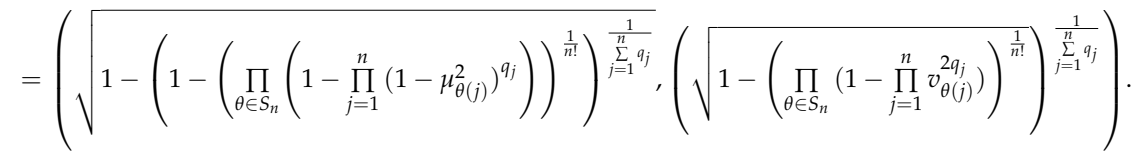

Theorem 8. The Pythagorean fuzzy weighted geometric averaging operator [6] is a special case of the PFDWMM operator.

Proof. When $Q=(1,0, \cdots, 0)$,

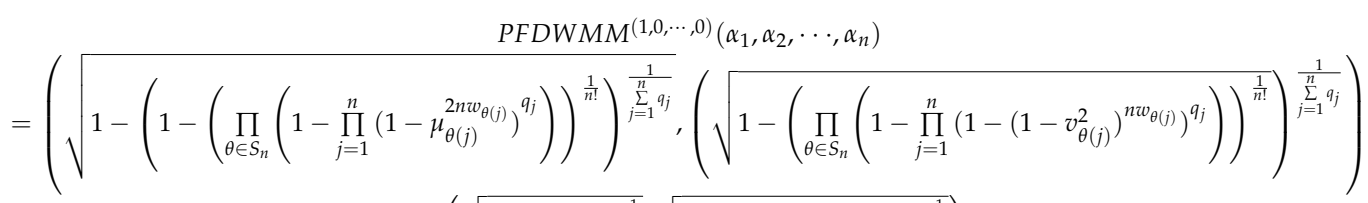

$$
\begin{aligned}
& =\left(\sqrt{\left(\prod_{j=1}^{n} \mu_{j}^{2 n w_{j}}\right)^{\frac{1}{n}}}, \sqrt{1-\left(\prod_{j=1}^{n}\left(1-v_{j}^{2}\right)^{n v v_{j}}\right)^{\frac{1}{n}}}\right) \\
& =\left(\prod_{j=1}^{n} \mu_{P_{j}}^{w_{j}} \sqrt{1-\prod_{j=1}^{n}\left(1-v_{P_{j}}^{2}\right)^{z v_{j}}}\right) .
\end{aligned}
$$

\section{New Approach to MCGDM with Pythagorean Fuzzy Information}

In this section, we propose a new MCGDM method under the Pythagorean fuzzy environment based on the PFWMM operator or PFDWMM operator. A typical MCGDM problem with Pythagorean fuzzy information can be described as follows. Let $A=\left\{A_{1}, A_{2}, \cdots, A_{m}\right\}$ be a discrete set of alternatives, and $C=\left\{C_{1}, C_{2}, \cdots, C_{n}\right\}$ be a finite set of criteria with the weight vector is $w=\left\{w_{1}, w_{2}, \cdots, w_{n}\right\}$, satisfying $w_{j} \in[0,1](j=1,2, \cdots, n)$ and $\sum_{j=1}^{n} w_{j}=1$. Assume that $E=\left\{E_{1}, E_{2}, \cdots, E_{p}\right\}$ be a finite set of experts with the weight vector is $\eta=\left\{\eta_{1}, \eta_{2}, \cdots, \eta_{p}\right\}$, satisfying $\eta_{k} \in[0,1](k=1,2, \cdots, p)$ and $\sum_{k=1}^{p} \eta_{k}=1$. The evaluation information of alternative $A_{i}(i=1,2, \cdots, m)$ with respect to criteria $C_{j}(j=1,2, \cdots, n)$ provided by the expert $E_{k}(k=1,2, \cdots, p)$ can be denoted as $\alpha_{i j}^{k}=\left(\mu_{P_{i j}}^{k}, v_{P_{i j}}^{k}\right)$, where $\alpha_{i j}^{k}$ is a PFN. Therefore, the Pythagorean fuzzy evaluation matrix $R^{k}=\left(\alpha_{i j}^{k}\right)_{m \times n}$ provided by the expert $E_{k}$ is obtained.

In what follows, a novel approach based on the PFWMM operator or PFDWMM operator is proposed to solve the MCGDM problem with Pythagorean fuzzy information, and the detailed steps are depicted as follows.

Step 1: Generally, there are two types of criteria, i.e., benefit criterion and cost criterion. Therefore, the Pythagorean fuzzy evaluation matrix should be normalized by

$$
\alpha_{i j}^{k}=\left\{\begin{array}{c}
\left(\mu_{P_{i j} j^{\prime}}^{k}, v_{P_{i j}}^{k}\right), \text { for benefit criterion. } \\
\left(v_{P_{i j}}^{k}, \mu_{P_{i j}}^{k}\right), \text { for cos t criterion. }
\end{array}\right.
$$


Step 2: Construct the group decision matrix $R=\left(\alpha_{i j}\right)_{m \times n}$ by applying the PFWMM operator or PFDWMM operators to aggregate all individual evaluation matrix $R^{k}$.

$$
\alpha_{i j}=\operatorname{PFWMM}\left(\alpha_{i j}^{1}, \alpha_{i j}^{2}, \cdots, \alpha_{i j}^{p}\right), \alpha_{i j}=\operatorname{PFDWMM}\left(\alpha_{i j}^{1}, \alpha_{i j}^{2}, \cdots, \alpha_{i j}^{p}\right) .
$$

Step 3: Calculate the comprehensive evaluation value $\alpha_{i}$ by using the PFWMM or PFDWMM operators to aggregate all the performance values of alternative with regard to each criterion.

$$
\alpha_{i}=\operatorname{PFWMM}\left(\alpha_{i 1}, \alpha_{i 2}, \cdots, \alpha_{i n}\right), \alpha_{i}=\operatorname{PFDWMM}\left(\alpha_{i 1}, \alpha_{i 2}, \cdots, \alpha_{i n}\right) .
$$

Step 4: Determine the priority of alternatives according to the score value $S\left(\alpha_{i}\right)$.

\section{An Example}

To validate the effectiveness and feasibility of the proposed method, we adopt a numerical example that is about the selection decision of enterprise resource planning (ERP) system. An enterprise wants to select a suitable ERP system to improve the competitive capability of the company. In order to make a scientific decision, three experts are selected to form the expert team, denoted as $E=\left\{E_{1}, E_{2}, E_{3}\right\}$, where $E_{1}$ is a $C I O, E_{2}$ and $E_{3}$ are two senior representatives from the user department. Suppose that the importance of experts is equal, namely, $\eta=(1 / 3,1 / 3,1 / 3)^{T}$. Through analyzing the ERP system, the expert team determines the assessment criteria including function and technology $\left(C_{1}\right)$, strategic fitness $\left(C_{2}\right)$, vendor ability $\left(C_{3}\right)$, and vendor reputation $\left(C_{4}\right)$. According to the existing experience and knowledge, the weight vector of the criteria is assigned by experts as $w=(0.2,0.1,0.3,0.4)^{T}$. Five potential ERP systems $A=\left\{A_{1}, A_{2}, A_{3}, A_{4}, A_{5}\right\}$ are chosen by the expert team as candidates. Subsequently, the experts adopt PFNs to provide the assessment information of the alternatives with regard to each criterion. The Pythagorean fuzzy decision matrices are provided by three experts, shown in Tables 1-3, respectively. In what follows, we apply the proposed method to obtain the best ERP system for the enterprise.

Table 1. The Pythagorean fuzzy decision matrix provided by the $E_{1}$.

\begin{tabular}{ccccc}
\hline Alternatives & $\boldsymbol{C}_{\mathbf{1}}$ & $\boldsymbol{C}_{\mathbf{2}}$ & $\boldsymbol{C}_{\mathbf{3}}$ & $\boldsymbol{C}_{\mathbf{4}}$ \\
\hline$A_{1}$ & $(0.4,0.8)$ & $(0.8,0.6)$ & $(0.6,0.7)$ & $(0.3,0.8)$ \\
$A_{2}$ & $(0.7,0.5)$ & $(0.8,0.4)$ & $(0.8,0.5)$ & $(0.3,0.6)$ \\
$A_{3}$ & $(0.3,0.4)$ & $(0.3,0.7)$ & $(0.7,0.4)$ & $(0.6,0.4)$ \\
$A_{4}$ & $(0.6,0.6)$ & $(0.7,0.5)$ & $(0.7,0.2)$ & $(0.4,0.6)$ \\
$A_{5}$ & $(0.5,0.7)$ & $(0.6,0.4)$ & $(0.9,0.3)$ & $(0.6,0.7)$ \\
\hline
\end{tabular}

Table 2. The Pythagorean fuzzy decision matrix provided by the $E_{2}$.

\begin{tabular}{ccccc}
\hline Alternatives & $C_{1}$ & $C_{\mathbf{2}}$ & $C_{3}$ & $C_{4}$ \\
\hline$A_{1}$ & $(0.3,0.9)$ & $(0.7,0.6)$ & $(0.5,0.8)$ & $(0.3,0.6)$ \\
$A_{2}$ & $(0.7,0.4)$ & $(0.9,0.2)$ & $(0.8,0.1)$ & $(0.3,0.5)$ \\
$A_{3}$ & $(0.3,0.6)$ & $(0.7,0.7)$ & $(0.7,0.6)$ & $(0.4,0.4)$ \\
$A_{4}$ & $(0.4,0.8)$ & $(0.7,0.5)$ & $(0.6,0.2)$ & $(0.4,0.7)$ \\
$A_{5}$ & $(0.2,0.7)$ & $(0.8,0.2)$ & $(0.8,0.4)$ & $(0.6,0.6)$ \\
\hline
\end{tabular}

Table 3. The Pythagorean fuzzy decision matrix provided by the $E_{3}$.

\begin{tabular}{ccccc}
\hline Alternatives & $\boldsymbol{C}_{\mathbf{1}}$ & $\boldsymbol{C}_{\mathbf{2}}$ & $\boldsymbol{C}_{\mathbf{3}}$ & $\boldsymbol{C}_{\mathbf{4}}$ \\
\hline$A_{1}$ & $(0.6,0.8)$ & $(0.7,0.6)$ & $(0.5,0.8)$ & $(0.5,0.5)$ \\
$A_{2}$ & $(0.6,0.5)$ & $(0.9,0.2)$ & $(0.8,0.1)$ & $(0.3,0.5)$ \\
$A_{3}$ & $(0.4,0.7)$ & $(0.7,0.5)$ & $(0.6,0.1)$ & $(0.2,0.9)$ \\
$A_{4}$ & $(0.2,0.9)$ & $(0.5,0.6)$ & $(0.6,0.2)$ & $(0.1,0.6)$ \\
$A_{5}$ & $(0.1,0.6)$ & $(0.8,0.2)$ & $(0.9,0.2)$ & $(0.6,0.5)$ \\
\hline
\end{tabular}




\subsection{Implementation of the Proposed Method}

To obtain the best ERP system, the computation steps are shown in the following:

Step 1: The criterion value of the ERP system does not require normalization because all the criteria are benefit type.

Step 2: Based on the individual evaluation matrix $R^{k}(k=1,2,3)$, we employ the PFWMM operator or PFDWMM operator (Suppose $Q=(1,1,1)$ ) to obtain the group decision matrix $R$, and the results are shown in Tables 4 and 5 , respectively.

Table 4. The collective decision matrix obtained by the PFWMM operator.

\begin{tabular}{ccccc}
\hline Alternatives & $\boldsymbol{C}_{\mathbf{1}}$ & $\boldsymbol{C}_{\mathbf{2}}$ & $\boldsymbol{C}_{\mathbf{3}}$ & $\boldsymbol{C}_{\boldsymbol{4}}$ \\
\hline$A_{1}$ & $(0.4160,0.8421)$ & $(0.7319,0.6000)$ & $(0.5313,0.7718)$ & $(0.3557,0.6656)$ \\
$A_{2}$ & $(0.6649,0.4702)$ & $(0.8653,0.2860)$ & $(0.8000,0.3123)$ & $(0.3000,0.5372)$ \\
$A_{3}$ & $(0.3302,0.5919)$ & $(0.5278,0.6481)$ & $(0.6649,0.4354)$ & $(0.3634,0.6987)$ \\
$A_{4}$ & $(0.3634,0.8047)$ & $(0.6257,0.5372)$ & $(0.6316,0.2000)$ & $(0.2520,0.6377)$ \\
$A_{5}$ & $(0.2154,0.6707)$ & $(0.7268,0.2860)$ & $(0.8653,0.3131)$ & $(0.6000,0.6119)$ \\
\hline
\end{tabular}

Table 5. The collective decision matrix obtained by the PFDWMM operator.

\begin{tabular}{ccccc}
\hline Alternatives & $\boldsymbol{C}_{\mathbf{1}}$ & $\boldsymbol{C}_{\mathbf{2}}$ & $\boldsymbol{C}_{\mathbf{3}}$ & $\boldsymbol{C}_{\boldsymbol{4}}$ \\
\hline$A_{1}$ & $(0.4605,0.8320)$ & $(0.7389,0.6000)$ & $(0.5372,0.7652)$ & $(0.3832,0.6214)$ \\
$A_{2}$ & $(0.6707,0.4642)$ & $(0.8746,0.2520)$ & $(0.8000,0.1710)$ & $(0.3000,0.5313)$ \\
$A_{3}$ & $(0.3376,0.5518)$ & $(0.6176,0.6257)$ & $(0.6707,0.2884)$ & $(0.4448,0.5241)$ \\
$A_{4}$ & $(0.4448,0.7560)$ & $(0.6481,0.5313)$ & $(0.6377,0.2000)$ & $(0.3357,0.6316)$ \\
$A_{5}$ & $(0.3267,0.6649)$ & $(0.7509,0.2520)$ & $(0.8746,0.2884)$ & $(0.6000,0.5944)$ \\
\hline
\end{tabular}

Step 3: With the aid of the PFWMM operator or PFDWMM operator (suppose $Q=(1,1,1,1)$ ), the comprehensive evaluation value $\alpha_{i}$ of the ERP system can be obtained. The calculated results are shown in Table 6.

Table 6. The comprehensive evaluation value by PFWMM and PFDWMM operators.

\begin{tabular}{cccccc}
\hline Operator & $A_{\mathbf{1}}$ & $A_{\mathbf{2}}$ & $A_{\mathbf{3}}$ & $A_{\mathbf{4}}$ & $A_{\mathbf{5}}$ \\
\hline PFWMM & $(0.4700,0.7707)$ & $(0.5948,0.4751)$ & $(0.4150,0.6672)$ & $(0.5015,0.5599)$ & $(0.4269,0.6635)$ \\
PFDWMM & $(0.6483,0.6583)$ & $(0.7915,0.3001)$ & $(0.6264,0.4482)$ & $(0.7478,0.3847)$ & $(0.6142,0.4285)$ \\
\hline
\end{tabular}

Step 4: Calculate the scores of the comprehensive evaluation value $\alpha_{i}$ of each ERP system (results shown in Table 7). The ranking of the ERP systems can be obtained according to the scores in descending order. The obtained rankings are shown in Table 7.

Table 7. The score values and the ranking results of five alternatives by two operators.

\begin{tabular}{ccccccc}
\hline Operator & $S\left(\boldsymbol{\alpha}_{\mathbf{1}}\right)$ & $S\left(\boldsymbol{\alpha}_{\mathbf{2}}\right)$ & $S\left(\boldsymbol{\alpha}_{\mathbf{3}}\right)$ & $S\left(\boldsymbol{\alpha}_{\mathbf{4}}\right)$ & $S\left(\boldsymbol{\alpha}_{\mathbf{5}}\right)$ & Ranking Order \\
\hline PFWMM & 0.3135 & 0.5640 & 0.3710 & 0.3568 & 0.4690 & $A_{2} \succ A_{5} \succ A_{3} \succ A_{4} \succ A_{1}$ \\
PFDWMM & 0.4935 & 0.7682 & 0.5968 & 0.5957 & 0.7056 & $A_{2} \succ A_{5} \succ A_{3} \succ A_{4} \succ A_{1}$ \\
\hline
\end{tabular}

Based on the ranking results in Table 7, we know that the ranking order obtained by the PFWMM operator and PFDWMM operator are the same, and the best ERP system is $A_{2}$.

To further demonstrate the effectiveness and applicability of the presented approach, we employ the proposed method to solve two practical MCDM problems concerned with the investment decision respecting Internet stocks [11] and investment decision respecting R\&D projects [11]. This paper refers to individual decision-making, while these two investment decision problems are group 
decision-making problems. Hence, the implementation of the presented approach is performed based on the comprehensive evaluation matrix that is obtained by employing the PFWA operator to aggregate the individual evaluation matrix.

The investment decision problem of Internet stocks involves four Internet stocks $\left(A_{1}, A_{2}, A_{3}, A_{4}\right)$ and three benefit criteria $\left(C_{1}, C_{2}, C_{3}\right)$, and the weight vector of the criterion is $w=(0.5,0.2,0.3)$. The comprehensive evaluation matrix of Internet stocks with respect to each criterion is shown in Table 8. The obtained results and ranking orders are shown in Table 9. From Table 9, we can see that the ranking order obtained by the proposed method and algorithm 1 is totally identical. The best and worst Internet stocks are $A_{1}$ and $A_{4}$, respectively.

Table 8. The collective evaluation matrix of Internet stocks regarding each criterion [11].

\begin{tabular}{cccc}
\hline Alternatives & $\boldsymbol{C}_{\mathbf{1}}$ & $\boldsymbol{C}_{\mathbf{2}}$ & $\boldsymbol{C}_{\mathbf{3}}$ \\
\hline$A_{1}$ & $(0.77,0.19)$ & $(0.88,0.18)$ & $(0.77,0.17)$ \\
$A_{2}$ & $(0.61,0.67)$ & $(0.51,0.56)$ & $(0.67,0.18)$ \\
$A_{3}$ & $(0.68,0.27)$ & $(0.70,0.51)$ & $(0.67,0.48)$ \\
$A_{4}$ & $(0.66,0.62)$ & $(0.56,0.67)$ & $(0.56,0.36)$ \\
weight & 0.5 & 0.2 & 0.3 \\
\hline
\end{tabular}

Table 9. The score values and ranking results of four Internet stocks.

\begin{tabular}{cllllc}
\hline Operator & $S\left(A_{\mathbf{1}}\right)$ & $S\left(A_{\mathbf{2}}\right)$ & $S\left(A_{\mathbf{3}}\right)$ & $S\left(A_{\mathbf{4}}\right)$ & Ranking Order \\
\hline Algorithm 1 [11] & 0.5944 & 0.1183 & 0.3195 & 0.0807 & $A_{1} \succ A_{3} \succ A_{2} \succ A_{4}$ \\
PFWMM & 0.7818 & 0.5098 & 0.5865 & 0.4737 & $A_{1} \succ A_{3} \succ A_{2} \succ A_{4}$ \\
PFDWMM & 0.8313 & 0.6223 & 0.6741 & 0.5614 & $A_{1} \succ A_{3} \succ A_{2} \succ A_{4}$ \\
\hline
\end{tabular}

The investment decision problem of the $R \& D$ project includes three potential $R \& D$ projects $\left(A_{1}, A_{2}, A_{3}\right)$ and five benefit criteria $\left(C_{1}, C_{2}, C_{3}, C_{4}, C_{5}\right)$; the weight vector of the criterion is $w=(0.2,0.1,0.3,0.15,0.25)$. The comprehensive evaluation matrix of $R \& D$ projects based on each criterion is shown in Table 10, and the ranking index and ranking results of three R\&D projects are presented in Table 11. From Table 11, we know that the ranking order of R\&D projects obtained in the developed approach is the same as that determined by algorithm 2 . The preferred R\&D project is $A_{1}$, and the worst one is $A_{3}$.

Table 10. The collective evaluation matrix of R\&D project regarding each criterion [11].

\begin{tabular}{cccccc}
\hline Alternatives & $\boldsymbol{C}_{\mathbf{1}}$ & $\boldsymbol{C}_{\mathbf{2}}$ & $\boldsymbol{C}_{\mathbf{3}}$ & $\boldsymbol{C}_{\mathbf{4}}$ & $\boldsymbol{C}_{\mathbf{5}}$ \\
\hline$A_{1}$ & $(0.77,0.21)$ & $(0.71,0.18)$ & $(0.77,0.17)$ & $(0.75,0.10)$ & $(0.76,0.20)$ \\
$A_{2}$ & $(0.59,0.64)$ & $(0.53,0.46)$ & $(0.67,0.29)$ & $(0.45,0.65)$ & $(0.80,0.24)$ \\
$A_{3}$ & $(0.68,0.34)$ & $(0.68,0.51)$ & $(0.67,0.51)$ & $(0.80,0.45)$ & $(0.45,0.77)$ \\
weight & 0.2 & 0.1 & 0.3 & 0.15 & 0.25 \\
\hline
\end{tabular}

Table 11. The ranking index and ranking results of three R\&D projects.

\begin{tabular}{ccccc}
\hline Operator & $S\left(\boldsymbol{A}_{\mathbf{1}}\right)$ & $S\left(\boldsymbol{A}_{\mathbf{2}}\right)$ & $S\left(A_{\mathbf{3}}\right)$ & Ranking Order \\
\hline Algorithm 2 [11] & 0.9083 & -1.1927 & -2.2731 & $A_{1} \succ A_{2} \succ A_{3}$ \\
PFWMM & 0.7331 & 0.5008 & 0.5279 & $A_{1} \succ A_{2} \succ A_{3}$ \\
PFDWMM & 0.5931 & 0.4230 & 0.3449 & $A_{1} \succ A_{2} \succ A_{3}$ \\
\hline
\end{tabular}

Based on the above analysis on two investment decision problems, we can conclude that the method presented in this paper is effective and feasible. 


\subsection{Sensitivity Analysis}

To illustrate the influence of different values of parameter vector $Q$, we change the values of parameter vector $Q$ in our proposed method to rank the alternatives. The results are shown in Table 12.

Table 12. Ranking order of alternatives determined by different parameter vector $Q$.

\begin{tabular}{cll}
\hline Parameter Vector $Q$ & Ranking Order by PFWMM & Ranking Order by PFDWMM \\
\hline$Q=(3,0,0,0)$ & $A_{5} \succ A_{2} \succ A_{4} \succ A_{3} \succ A_{1}$ & $A_{5} \succ A_{3} \succ A_{2} \succ A_{4} \succ A_{1}$ \\
$Q=(2,0,0,0)$ & $A_{5} \succ A_{2} \succ A_{4} \succ A_{3} \succ A_{1}$ & $A_{5} \succ A_{2} \succ A_{3} \succ A_{4} \succ A_{1}$ \\
$Q=(1,0,0,0)$ & $A_{2} \succ A_{5} \succ A_{4} \succ A_{3} \succ A_{1}$ & $A_{2} \succ A_{5} \succ A_{3} \succ A_{4} \succ A_{1}$ \\
$Q=(1,1,0,0)$ & $A_{2} \succ A_{5} \succ A_{3} \succ A_{4} \succ A_{1}$ & $A_{2} \succ A_{5} \succ A_{3} \succ A_{4} \succ A_{1}$ \\
$Q=(1,1,1,0)$ & $A_{2} \succ A_{5} \succ A_{3} \succ A_{4} \succ A_{1}$ & $A_{2} \succ A_{5} \succ A_{3} \succ A_{4} \succ A_{1}$ \\
$Q=(1,1,1,1)$ & $A_{2} \succ A_{5} \succ A_{3} \succ A_{4} \succ A_{1}$ & $A_{2} \succ A_{5} \succ A_{3} \succ A_{4} \succ A_{1}$ \\
$Q=(0.25,0.25,0.25,0.25)$ & $A_{2} \succ A_{5} \succ A_{3} \succ A_{4} \succ A_{1}$ & $A_{2} \succ A_{5} \succ A_{3} \succ A_{4} \succ A_{1}$ \\
\hline
\end{tabular}

From Table 12, we know the ERP systems obtained with different parameter vectors $Q$ are slightly different. The main reason is that the PFWMM operator highlights the impact of overall arguments, but the PFDWMM operator emphasizes the role of individual arguments. When $Q=(1,0,0,0)$, it is worth noting that the PFWMM operator and PFDWMM operator will reduce to Pythagorean fuzzy weighted averaging operator and Pythagorean fuzzy weighted geometric operator, respectively. In addition, from Figures 1 and 2 we can make the following conclusions. For the PFWMM operator, when the parameter vector $Q$ has only one real number and the rest are 0 , we discover that the larger the real number of parameter vector $Q$, the greater the value of the score function will become. The more interdependent relationships of criteria we consider, the smaller the score function will become. Nevertheless, for the PFDWMM operator, the conclusion is just the opposite: that is, the greater the real number of parameter vector $Q$, the smaller the value of the score function will become. The more relationships between attributes we consider, the larger the value of the score function will become. Therefore, the experts can select different values of parameter vector $Q$ based on different risk preferences.

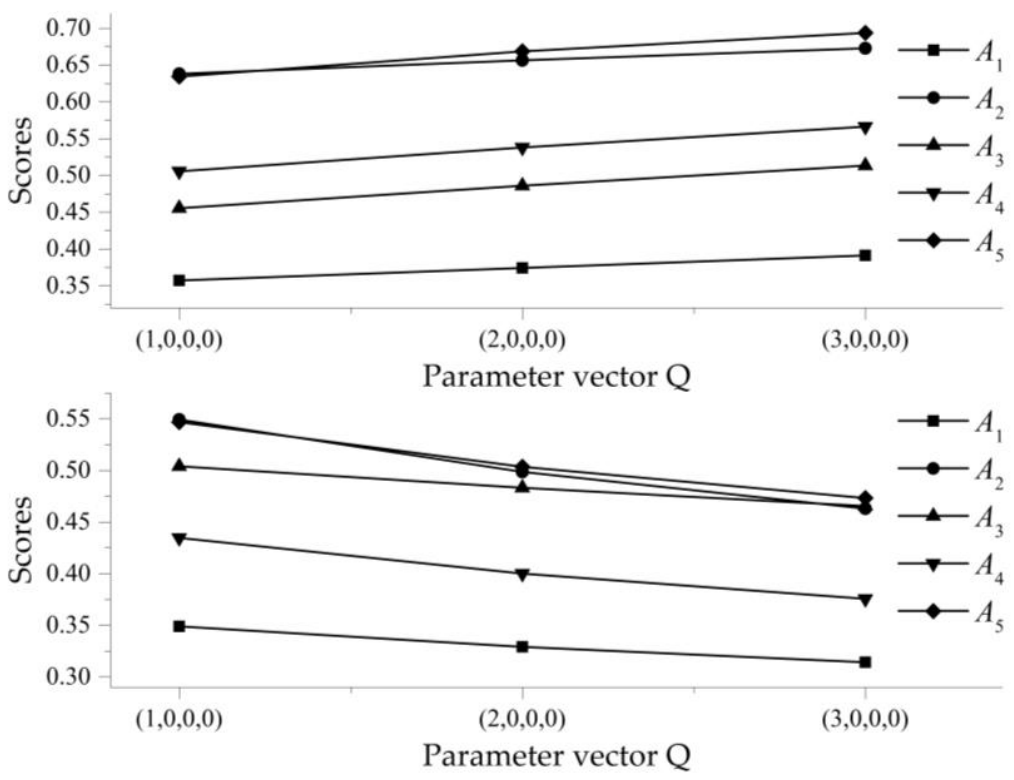

Figure 1. Scores of alternatives for different parameter vector $Q$. 


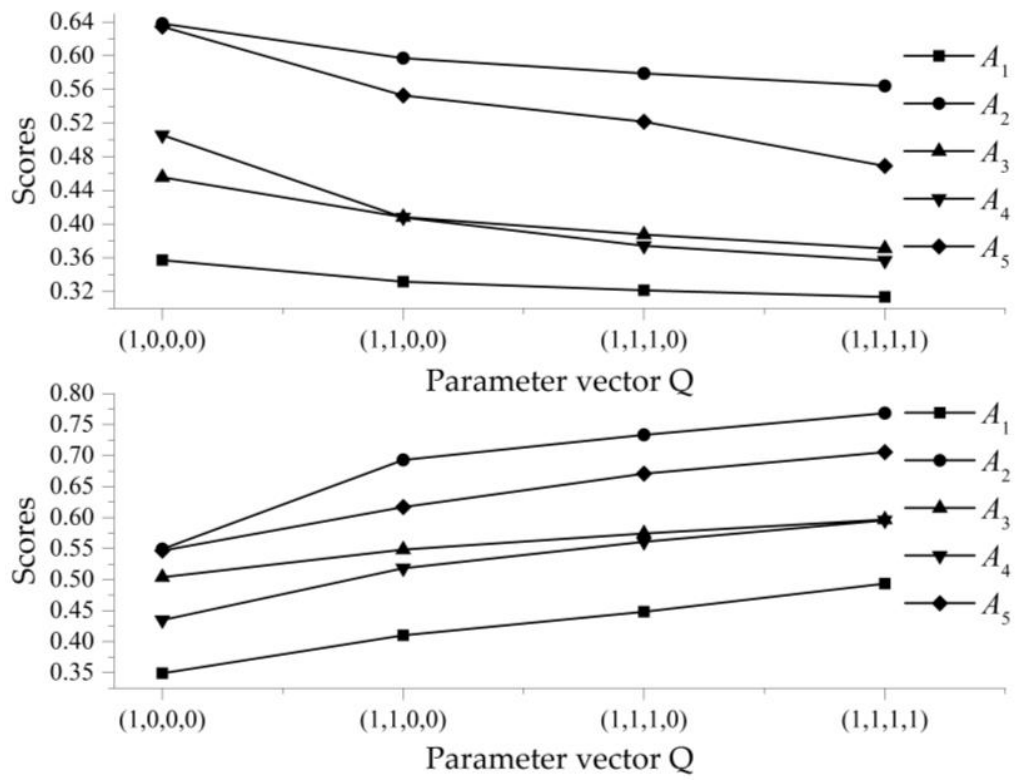

Figure 2. Scores of alternatives for interrelationship of different arguments.

\subsection{Comparative Analysis}

In order to further verify the validity and illustrate the advantage of the proposed approach, we compare our developed method with other existing MCGDM methods including the Pythagorean fuzzy weighted averaging (PFWA) operator and Pythagorean fuzzy weighted geometric (PFWG) operator [6], the symmetric Pythagorean fuzzy weighted averaging (SPFWA) operator and symmetric Pythagorean fuzzy weighted geometric (SPFWG) operator [6], the Pythagorean fuzzy weighted geometric Bonferroni mean (PFWGBM) operator [14], and the Pythagorean fuzzy weighted Maclaurin symmetric mean (PFWMSM) operator [25]. The ranking results are shown in Table 13.

Table 13. Ranking order of alternatives obtained by different methods.

\begin{tabular}{ccc}
\hline Operator & Parameter & Ranking Order \\
\hline PFWA & No & $A_{2} \succ A_{5} \succ A_{3} \succ A_{4} \succ A_{1}$ \\
PFWG & No & $A_{2} \succ A_{5} \succ A_{4} \succ A_{3} \succ A_{1}$ \\
SPFWA & No & $A_{2} \succ A_{5} \succ A_{3} \succ A_{4} \succ A_{1}$ \\
SPFWG & No & $A_{2} \succ A_{5} \succ A_{3} \succ A_{4} \succ A_{1}$ \\
PFWGBM & $p=q=1$ & $A_{2} \succ A_{5} \succ A_{3} \succ A_{4} \succ A_{1}$ \\
PFWMSM & $k=2$ & $A_{2} \succ A_{5} \succ A_{3} \succ A_{4} \succ A_{1}$ \\
PFWMM & $Q=(1,1,1,1)$ & $A_{2} \succ A_{5} \succ A_{3} \succ A_{4} \succ A_{1}$ \\
PFDWMM & $Q=(1,1,1,1)$ & $A_{2} \succ A_{5} \succ A_{3} \succ A_{4} \succ A_{1}$ \\
\hline
\end{tabular}

As we can see from Table 13, the ranking order of the ERP systems by the PFWG operator is slightly different with the other methods, but the best and worst ERP systems are $A_{2}$ and $A_{1}$, respectively. This verifies that the PFWMM and PFDWMM operators we developed are reasonable and valid for MCGDM problems with Pythagorean fuzzy information.

In what follows, the comparisons of proposed approaches and the other methods with regard to some characteristics are shown in Table 14. In light of Table 14, some conclusions are summarized as follows:

(1) The two methods developed by $\mathrm{Ma}$ and $\mathrm{Xu}$ [6] aggregate fuzzy information easily. The drawbacks of $\mathrm{Ma}$ and $\mathrm{Xu}$ 's method are they assume that the input arguments are not correlated, that is, they fail to consider the relationships between the input arguments. Nevertheless, our developed operators can capture the correlations among all the input arguments, 
and fuse fuzzy information more flexibly by the parameter vector. Furthermore, the PFWA and PFWG operators are a special case of PFWMM and PFDWMM operators, respectively, when the parameter vector $Q=(1,0,0,0)$. Therefore, our developed approaches are more general and flexible comparing with that proposed by Ma and $\mathrm{Xu}$.

(2) The primary advantage of our proposed operators is that they can capture the relationships between the multi-input arguments, while the method proposed by Liang et al. [14] can only deal with a correlation between any two input arguments. In reality, interdependent relationships may exist between more than two input arguments. Apparently, the PFWGBM operator is unable to handle this situation because it only captures the relationship between any two arguments. Furthermore, we also find that the PFDMM operator can transform into the PFGBM operator when the parameter vector is set to $Q=(1,1,0, \cdots, 0)$. Therefore, our approach can overcome the weakness of the PFWGBM operator because our operators can deal with any number of input arguments being interdependent.

(3) Compared with our developed operators, although the PFWMSM operator can also deal with relationships between multi-input arguments, our methods can provide a more flexible information aggregation process through setting different parameter vector $Q$. Similarly, we can obtain the PFMSM operator when the parameter vector is set to $Q=(\underbrace{1,1, \cdots, 1}_{k}, \underbrace{0,0, \cdots, 0}_{n-k})$. Thus, our developed operators are more general.

In short, based on the above comparisons and discussion, we can conclude that there are advantages to the PFWMM operator and PFDWMM operator compared with the existing other operators, including (a) they can capture the relationships between the multi-input arguments; and (b) they are more robust and it is more convenient to fuse the Pythagorean fuzzy information by the parameter vector $Q$.

Table 14. A comparison of the different approaches.

\begin{tabular}{cccc}
\hline Approaches & $\begin{array}{c}\text { Captures Correlation of } \\
\text { Two Criteria }\end{array}$ & $\begin{array}{c}\text { Captures Correlation of } \\
\text { Multiple Criteria }\end{array}$ & $\begin{array}{c}\text { Makes Method Flexible } \\
\text { by the Parameter Vector }\end{array}$ \\
\hline PFWA & No & No & No \\
PFWG & No & No & No \\
SPFWA & No & No & No \\
SPFWG & No & No & No \\
PFWGBM & Yes & No & No \\
PFWMSM & Yes & Yes & No \\
PFWMM & Yes & Yes & Yes \\
PFDWMM & Yes & Yes & Yes \\
\hline
\end{tabular}

\section{Conclusions}

In recent years, a number of researchers have developed aggregation operators under various fuzzy environments and applied these to solve different decision-making problems. However, these aggregation operators have some drawbacks in actual applications, such as being unable to reflect the correlation of all input arguments. The MM operator has an apparent advantage in that it can deal with the relationships between all the input arguments according to the parameter vector $Q$. Motivated by the ideal characteristic of the MM operator, in this paper we extended the MM operator into the Pythagorean fuzzy environment to deal with MCGDM problems with relationships between any number of arguments. We proposed some aggregation operators, including the PFMM operator, PFWMM operator, PFDMM operator, and PFDWMM operator. Then, some desirable properties and special cases of the proposed operators were investigated and discussed in detail. In addition, we have used the PFWMM and PFDWMM operators to present two methods to solve MCGDM problems with PFNs. Finally, we gave an example to demonstrate the effectiveness and feasibility of the presented methods through comparing with other existing approaches. 
In future research, it is necessary to verify the validity of the proposed methods by solving other MCGDM problems such as supplier selection, risk assessment, and environment evaluation. Moreover, based on the prominent characteristics of the MM operator, we shall extend the MM operator into other fuzzy contexts such as the interval-valued 2-tuple linguistic environment, the interval-valued Pythagorean fuzzy environment, and the triangle intuitionistic fuzzy context.

Author Contributions: Conceptualization and Methodology, J.Z. and Y.L.; Data Curation, J.Z.; Writing-Original Draft Preparation, J.Z.; Writing—Review\& Editing, J.Z. and Y.L.; Visualization, J.Z.; Supervision, Y.L.

Funding: This research was funded by the National Natural Science Foundation of China (No. 71371156) and the Doctoral Innovation Fund Program of Southwest Jiaotong University (D-CX201729).

Conflicts of Interest: The authors declare no conflict of interest.

\section{Abbreviations}

All abbreviations used in this paper:

$\begin{array}{ll}\text { BM } & \text { Bonferroni mean } \\ \text { ERP } & \text { Enterprise resource planning } \\ \text { FS } & \text { Fuzzy set } \\ \text { IFS } & \text { Intuitionistic fuzzy set } \\ \text { MCDM } & \text { Multi-criteria decision-making } \\ \text { MCGDM } & \text { Multi-criteria group decision-making } \\ \text { MM } & \text { Muirhead mean } \\ \text { MSM } & \text { Maclaurin symmetric mean } \\ \text { PFDMM } & \text { Pythagorean fuzzy dual Muirhead mean } \\ \text { PFDWMM } & \text { Pythagorean fuzzy dual weighted Muirhead mean } \\ \text { PFMM } & \text { Pythagorean fuzzy Muirhead mean } \\ \text { PFN } & \text { Pythagorean fuzzy number } \\ \text { PFS } & \text { Pythagorean fuzzy set } \\ \text { PFWA } & \text { Pythagorean fuzzy weighted averaging } \\ \text { PFWG } & \text { Pythagorean fuzzy weighted geometric } \\ \text { PFWGBM } & \text { Pythagorean fuzzy weighted geometric Bonferroni mean } \\ \text { PFWMM } & \text { Pythagorean fuzzy weighted Muirhead mean } \\ \text { PFWMSM } & \text { Pythagorean fuzzy weighted Maclaurin symmetric mean } \\ \text { SPFWA } & \text { Symmetric Pythagorean fuzzy weighted averaging } \\ \text { SPFWG } & \text { Symmetric Pythagorean fuzzy weighted geometric }\end{array}$

\section{References}

1. Zadeh, L.A. Fuzzy sets. Inf. Control 1965, 8, 338-353. [CrossRef]

2. Atanassov, K.T.; Rangasamy, P. Intuitionistic fuzzy sets. Fuzzy Sets Syst. 1986, 20, 87-96. [CrossRef]

3. Yager, R.R. Pythagorean fuzzy subsets. In Proceedings of the IFSA World Congress and NAFIPS Annual Meeting, Edmonton, AB, Canada, 24-28 June 2013; pp. 57-61.

4. Yager, R.R. Pythagorean membership grades in multicriteria decision making. IEEE Trans. Fuzzy Syst. 2014, 22, 958-965. [CrossRef]

5. Zhang, X.; Xu, Z. Extension of topsis to multiple criteria decision making with Pythagorean fuzzy sets. Int. J. Intell. Syst. 2015, 29, 1061-1078. [CrossRef]

6. Ma, Z.; Xu, Z. Symmetric Pythagorean fuzzy weighted geometric/averaging operators and their application in multicriteria decision-making problems. Int. J. Intell. Syst. 2016, 31, 1198-1219. [CrossRef]

7. Rahman, K.; Abdullah, S.; Ahmed, R.; Ullah, M. Pythagorean fuzzy Einstein weighted geometric aggregation operator and their application to multiple attribute group decision making. J. Intell. Fuzzy Syst. 2017, 33, 1-13. [CrossRef]

8. Garg, H. A new generalized Pythagorean fuzzy information aggregation using Einstein operations and its application to decision making. Int. J. Intell. Syst. 2016, 31, 886-920. [CrossRef] 
9. Garg, H. Confidence levels based Pythagorean fuzzy aggregation operators and its application to decision-making process. Comput. Math. Organ. Theory 2017, 23, 1-26. [CrossRef]

10. Zeng, S.; Chen, J.; Li, X. A hybrid method for Pythagorean fuzzy multiple-criteria decision making. Int. J. Inf. Technol. Decis. Mak. 2016, 15, 403-422. [CrossRef]

11. Peng, X.; Yang, Y. Pythagorean fuzzy choquet integral based mabac method for multiple attribute group decision making. Int. J. Intell. Syst. 2016, 31, 989-1020. [CrossRef]

12. Wei, G.; Lu, M. Pythagorean fuzzy power aggregation operators in multiple attribute decision making. Int. J. Intell. Syst. 2018, 33, 169-186. [CrossRef]

13. Liang, D.; Zhang, Y.; Xu, Z.; Darko, A.P. Pythagorean fuzzy bonferroni mean aggregation operator and its accelerative calculating algorithm with the multithreading. Int. J. Intell. Syst. 2018. [CrossRef]

14. Liang, D.; Xu, Z.; Darko, A.P. Projection model for fusing the information of Pythagorean fuzzy multicriteria group decision making based on geometric bonferroni mean. Int. J. Intell. Syst. 2017, 32, 966-987. [CrossRef]

15. Muirhead, R.F. Some methods applicable to identities and inequalities of symmetric algebraic functions of $\mathrm{n}$ letters. Proc. Edinb. Math. Soc. 1902, 21, 144-162. [CrossRef]

16. Bonferroni, C. Sulle medie multiple di potenze. Bollettino dell'Unione Matematica Italiana 1950, 5, $267-270$.

17. Detemple, D.W.; Robertson, J.M. On generalized symmetric means of two varibles. Angew. Chem. 1979, 47, 4638-4660.

18. Qin, J.; Liu, X. 2-tuple linguistic Muirhead mean operators for multiple attribute group decision making and its application to supplier selection. Kybernetes 2016, 45, 2-29. [CrossRef]

19. Liu, P.; You, X. Interval neutrosophic Muirhead mean operators and their application in multiple attribute group decision making. Int. J. Uncertain. Quantif. 2017, 7. [CrossRef]

20. Liu, P.; Li, D. Some Muirhead mean operators for intuitionistic fuzzy numbers and their applications to group decision making. PLoS ONE 2017, 12, e0168767. [CrossRef] [PubMed]

21. Liu, P.; Li, Y.; Zhang, M.; Zhang, L.; Zhao, J. Multiple-attribute decision-making method based on hesitant fuzzy linguistic Muirhead mean aggregation operators. Soft Comput. 2018, 1-12. [CrossRef]

22. Wang, J.; Zhang, R.; Zhu, X.; Xing, Y.; Buchmeister, B. Some hesitant fuzzy linguistic Muirhead means with their application to multi-attribute group decision making. Complexity 2018. [CrossRef]

23. Liu, P.; Teng, F. Some Muirhead mean operators for probabilistic linguistic term sets and their applications to multiple attribute decision-making. Appl. Soft Comput. 2018, 68, 396-431. [CrossRef]

24. Liu, H.C.; Li, Z.; Zhang, J.Q.; You, X.Y. A large group decision making approach for dependence assessment in human reliability analysis. Reliab. Eng. Syst. Saf. 2018, 176, 135-144. [CrossRef]

25. Wei, G.; Lu, M. Pythagorean fuzzy Maclaurin symmetric mean operators in multiple attribute decision making. Int. J. Intell. Syst. 2017. [CrossRef] 